\title{
WILEY-VCH
}

\section{Dynamics of anisotropic break-up in nanowires of FCC lattice structure}

\author{
Vyacheslav N. Gorshkov, Pooya Sareh*, Vladimir V. Tereshchuk, Arash Soleiman-Fallah
}

Prof. V. N. Gorshkov, V. V. Tereshchuk

National Technical University of Ukraine, Igor Sikorsky Kyiv Polytechnic Institute, 37

Prospect Peremogy, Kiev 03056, Ukraine

Dr. P. Sareh

School of Engineering, University of Liverpool, London Campus, 33 Finsbury Square, London EC2A 1AG, UK

E-mail: pooya.sareh@liverpool.ac.uk

Dr. A. Soleiman-Fallah

Department of Aeronautics, Imperial College London, South Kensington Campus, London SW7 2AZ, UK

Keywords: Plateau-Rayleigh instability, FCC lattice structure, anisotropy, break-up, nanowire, nanodrop

Abstract. Nanowires are known to be thermally unstable and may break up into chains of nanoparticles at temperatures below their melting point. This phenomenon is reminiscent of the well-known Rayleigh-Plateau instability of liquid jets, when periodic sausage-like perturbations of the jet surface increase with time. The corresponding nanowire/jet shape modifications must be followed by a decrease in total surface energy. However, unlike isotropic liquid jet surfaces, the realization of this abatement in the system with a strong lattice structure and physical dissimilarity of bounding facets, leads to specific features of nanowire break-up viz. significant variations in the size of nanodrops and the average distance between them, the formation of long-living dumbbells, and zigzag-like structures. Here, the physical mechanisms of such diversity are analyzed. Apart from scientific insights, the yielded results could be potentially useful in applications such as the development of optical waveguides based on ordered nanoparticles chains. 


\section{WILEY-VCH}

\section{Introduction}

Over the past two decades, much research has been dedicated to the investigation of characteristics of metal and semiconductor nanowires. This is primarily due to their beneficial physical and chemical properties which allow these structures to be used in biosensors ${ }^{[1-4]}$, solar cell technology ${ }^{[5]}$, waveguides ${ }^{[6]}$, and numerous other technological applications. For instance, in a recent study ${ }^{[7]}$, it was reported that silicon nanowires were successfully employed for sensing nanoparticles using resonant frequency shifts. Moreover, ultra-thin nanowires possess such unexpected properties as negative magnetoresistance and quantum conductance, which render them useful as building blocks of electronic circuits ${ }^{[8]}$.

Although ultra-thin nanowires possess numerous desirable qualities, there is one serious obstacle which stands in the way of their immediate implementation in technological applications. Nanowires are known to be thermally unstable and may break up into chains of nanoparticles at temperatures below their melting point. In previous studies ${ }^{[9-12]}$, it was reported that gold, copper, and platinum nanowires can break up at temperatures of approximately $300^{\circ} \mathrm{C}, 400^{\circ} \mathrm{C}$, and $600^{\circ} \mathrm{C}$, respectively, well below the melting points of metals from which they are synthesized (i.e. $1064^{\circ} \mathrm{C}$ for $\mathrm{Au}, 1085^{\circ} \mathrm{C}$ for $\mathrm{Cu}$ and $1768^{\circ} \mathrm{C}$ for $\mathrm{Pt})$. At the same time, silver nanowires ${ }^{[13]}$ turned out to be particularly unstable with a sub-zero break-up temperature of $-10^{\circ} \mathrm{C}$. Additionally, thermal instability causes significant changes in the surface morphology and physical properties of a nanowire even prior to the break-up. 


\section{WILEY-VCH}

According to previous studies ${ }^{[12,17,18]}$, the phenomenon of nanowire break-up takes place due to the existence of Plateau-Rayleigh ${ }^{[19,20,21]}$ instability which was first observed in liquid jets. The classical model by Nichols and Mullins ${ }^{[24]}$ for nanowires as well as the hydrodynamical model for liquid jets consider the mass transfer from regions of high surface curvature to regions with lower surface curvature. In the former case, this instability is driven by the surface diffusion of atoms, and in the latter, by internal flows. However, irrespective of the source of instability, sinusoidal perturbations in the radius have the maximal growth increment in time corresponding to the wavelength $\lambda_{R} \approx 9 r_{0}$, where $r_{0}$ is the initial radius of a nanowire/jet. The origin of this coincidence, as well as the value of $\lambda_{R}$ itself, can be explained on the basis of simple qualitative relations.

If the radius of a nanowire/jet is periodically modulated along its length by perturbations with wavelength $\lambda$ and amplitude $\varepsilon$ as

$$
r(x, t=0)=r_{0}-\left(\varepsilon^{2} / 4 r_{0}\right)-\varepsilon \cos (k x), \quad k=2 \pi / \lambda, \varepsilon \ll r_{0},
$$

then the change in its side surface, $S(\varepsilon)$, is equal to

$$
\Delta S(\varepsilon) \sim \varepsilon^{2}\left(\hat{k}^{2}-1\right) / \hat{k}, \hat{k}=2 \pi r_{0} / \lambda
$$

The radius presented in the form of Eq. 1 corresponds to preserving the nanowire/jet volume with an accuracy of $\sim \varepsilon^{4}$ that is needed for calculating the value, $\Delta S(\varepsilon)$, correctly. Then, the well-known relation for non-dissipative systems, i.e. $m_{\lambda / 2} \ddot{\Delta}_{c m} \sim-\partial \Delta E_{\text {surf }} / \partial \Delta_{c m}$, (where $m_{\lambda / 2} \sim 1 / \hat{k}$ is the moving mass contained in a half-wavelength segment, $\Delta_{c m} \sim \varepsilon(t) / \hat{k}$ is the 
corresponding displacement of the center of this mass and $\Delta E_{\text {surf }}=\sigma \Delta S(\varepsilon)$ is the change in the surface energy) results in the common relation for both cases under consideration

$$
\left(\ddot{\varepsilon}(t) / \widehat{k}^{2}\right) \sim \varepsilon\left(1-\widehat{k}^{2}\right) \sim-\partial \Delta E_{\text {surf }} / \partial \Delta_{c m} .
$$

Thus, the classical relation for growth increment, $\gamma$, is obtained on the basis of a unified framework: $\gamma^{2} \sim \hat{k}^{2}\left(1-\hat{k}^{2}\right)$. Its maximal value corresponds to the wave vector $\hat{k}_{R}^{2}=1 / 2$, i.e. $\lambda_{R} / r_{0} \approx 8.89$. The threshold of instability is $\widehat{k}^{2}<1$ or $\lambda>2 \pi r_{0}=\lambda_{\min }$.

It is to be noted that experimental results for inviscid liquid jets are in good agreement with Eq. (3), which evaluates the mean distance between the centers of neighboring droplets, $\Lambda$, as the wavelength of periodic perturbations with the maximal increment in time, $\Lambda \approx \lambda_{R}$ (i.e. $\Lambda / r_{0} \approx 8.9$ ). This means that the diameter, $D$, of the approximately spherical nanoparticles can be easily calculated to be $D=2\left(3 r_{0}^{2} \lambda_{R} / 4\right)^{1 / 3} \approx 3.8 r_{0}$. Consequently, the gaps between the neighboring nanodroplets is $\Delta=\lambda_{R}-D \approx 5.2 r_{0}$, i.e. $\Delta / D \approx 1.37$. However, sufficient ground does not exist for the measured value of the nanowire break-up parameter, $\Lambda / r_{0}$, to correspond to the classical value $\sim 9$. The reasons for this are as follows: (i) the density of surface energy, $\sigma$, is not isotropic; (ii) the surface perturbations of an initially cylindrical nanowire do not always correspond to Eq. (1); (iii) the possible nonlinear interaction of perturbation modes can strongly influence the nanowire break-up dynamics; and (iv) the classical model ${ }^{[24]}$ does not take into account the exchange of atoms between the nanowire surface and surrounding gas of free atoms thus cannot explain the fact that break-up parameter, $\Lambda / r_{0}$, increases at lower temperatures ${ }^{[26]}$. 


\section{WILEY-VCH}

Although the qualitative similarity between the break-up of nanowires and liquid jets $(\Lambda \approx$ $\left.9 r_{0}\right)$ is claimed in several studies ${ }^{[25,26]}$ according to the experimental results for Au presented in a study ${ }^{[25]}$, the gap $\Delta$ is noticeably wider than the size of the created nanoclusters $(\Delta / D \approx$ 2.6 instead of the classical value 1.4 ). Some discrepancies were also directly stated in an earlier work on Au-nanowires ${ }^{[27]}$, where for temperatures far below the melting point, the experimental values of $\Lambda / r_{0}$ were larger than those predicted by the continuum model ${ }^{[24]}$ and standard deviations of this parameter were typically of the order of $\pm 17-32 \%$ of the mean value. Moreover, many studies ${ }^{[25-27]}$ confirm that a nanowire initially breaks into extended fragments which eventually transform into a series of 'droplets'. It was experimentally observed ${ }^{[9,13,28,29]}$ that some fragments could form long-lived 2-3-body dumbbells, a phenomenon not observed in liquid jets. Other important features of the nanowire break-up are the strong influence of temperature ${ }^{[26]}$ and the orientation of a nanorod axis along different crystallographic axes, on the measured parameter $\Lambda / r_{0}{ }^{[27]}$. Such a variety of features in the nanowire break-up is expectable since even the dynamics of nanoscale liquid threads does not correspond to the classical physical model ${ }^{[31,32]}$, as analyzed by researchers ${ }^{[33,34,35]}$.

The goal of our study is to analyze, in detail, the physical factors that result in the diversity of observed break-up parameters. The results obtained using a kinetic Monte-Carlo model demonstrate that the statistical characteristics of these parameters are mainly determined by anisotropy of the surface energy density, $\sigma$, which, in its own turn, strongly depends on nanowire axis orientation. We have augmented the previous notions ${ }^{[22,23,46]}$ anent the breakup dynamics for nanowire orientations along the axes of low index direction ([100] and [111]) (see Supplementary Sections S1, S3, and S4). Such oriented nanowires mainly have rotational symmetries of orders 4 and 3, respectively and present, to some extent, 'isotropic' cases with the parameter of break-up being $\Lambda / r_{0} \approx 9$. However, even in these cases, diversity exists in 


\section{WILEY-VCH}

nanowire dynamics up to the first few ruptures and different levels of sensitivity to variations of the nanowire temperature are observed. At the initial stages of the break-up, periodic configurations, $r(x, t)$, with periods $\mathcal{L}<\lambda_{R}$ may dominate. Such structures mimic the Delaunay unduloids ${ }^{[36]}$ of the unstable equilibrium state. Excitation of these relatively shortwave perturbations occurs due to thermal roughening ${ }^{[37,38,39]}$ of crystal surfaces. (The free energy of the surface is minimized when establishing equilibrium with its own vapor, which is an important factor in the dynamics of nanoscale systems.)

The main part of the present study focuses on asymmetrical orientations [210], [211], [221], [320] and [321] that clearly demonstrate phenomena not inherent to quasi-isotropic systems (these orientations, marked by blue and olive colors, are presented in Fig. 1.) We show that the series of features observed in experiments as well as in our numerical simulations are based on the strong self-consistency between reshaping the nanowire and the 'driving force', $-\partial \Delta E_{\text {surf }} / \partial \Delta_{c m}$ (see. Eq. 3), precisely at the nonlinear stage. The locally formed necks tend to be bounded by the 'main' crystal facets with the lower density of surface energy, which, at the same time, must be coordinated with decreasing the total surface energy, $E_{\text {surf }}$, of the nanowire/nanorod. Orientation of each of the main facets relative to the nanowire axis determines both its global contribution to $E_{\text {surf }}$, and its local role in neck formation. The combination of these factors along with anisotropy of the surface energy density, the coefficient of surface diffusion, and rates of detachment-reattachment processes clarifies many seemingly contradictory experimental results previously mentioned. For instance, the parameter of break-up, $\Lambda / r_{0}$, can increase to up to 16 for the [320] nanowire orientation and the formation of $w$-like fractures in a nanowire during the break-up - an inexplicable phenomenon within the paradigm of Plateau-Rayleigh instability. The physical processes 
studied can be utilized in the controlled break-up of nanowires to produce nanocluster systems of specific morphology.

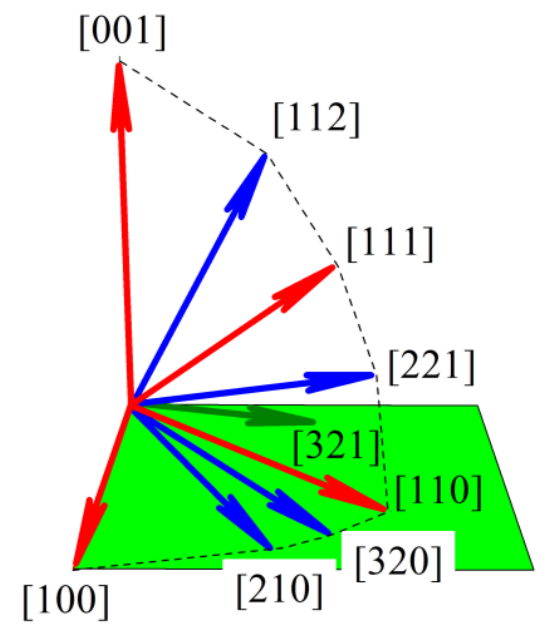

Fig. 1. Orientations of nanowires for which the break-up processes are analyzed in the present study.

\section{Theoretical Framework}

The Kinetic Monte-Carlo model used in the present study has been successfully applied in the mesoscale simulation of the processes of synthesis, dissolution, sintering, and growth of nanoparticles ${ }^{[30,40-46]}$, as well as surface growth for catalysis applications ${ }^{[49]}$. The model takes into account surface atom diffusion, besides the diffusion of atoms in the near-surface layer by means of detachment-reattachment processes. Some surface vacancies, surrounded by a large number of occupied sites of the crystal lattice, can go deep into the structure of the nanowire and cause the diffusion of internal atoms. Interestingly, despite being rare, such internal vacancies still cause noticeable mixing of atoms (see Supplementary Section S2). The basis of this numerical model is the assumption that the crystal structure of the nanowire is preserved at all stages of its dynamics. This is a sufficiently realistic model for the problem under consideration since a recent experimental study ${ }^{[47]}$ shows that even in the case of small gold nanoparticles ( 561 atoms \pm 14 with the size of $\sim 3.2 a$, where $a$ is the FCC lattice 


\section{WILEY-VCH}

spacing), the crystalline structure is preserved at high temperatures (up to $500^{\circ} \mathrm{C}$ ). In our numerical experiments, the radius of nanowires is taken to be $r_{0} \gtrsim 6 a$.

The system under consideration consists of a nanowire with FCC crystal structure and a diffuser 'gas' of free atoms, which can attach to as well as detach from the nanowire surface. This system is enclosed in a cylindrical 'container' with walls that reflect the free atoms. The radius of this container is at least one order of magnitude higher than the nanowire diameter.

The external 'gas' atoms diffuse by hopping with random angles of scattering and fixed length, $\ell$, of the hops: $\ell=1 / \sqrt{2}$ (the FCC lattice spacing, $a$, is taken here as the unit of length). If we also treat the time of scattering, $\tau$, as the unit of time, then the corresponding volume diffusion coefficient, $D_{v}$, is obtained as:

$$
D_{v}=\ell^{2} /(3 \tau)=1 / 6
$$

The bound atoms that constitute a nanowire occupy the FCC-lattice sites with coordination number $m_{c}=12$. Both the occupied and vacant FCC-sites, which surround the nanowire, are supposed to be centers of Wigner-Seitz cells. If a free atom randomly hops into the cell that encloses a near-surface vacancy (i.e., a vacancy that adjoins with occupied nearest-neighbor lattice sites), then this atom becomes absorbed by the crystal-lattice vacancy and registered as part of the nanostructure.

Each crystal-lattice atom can hop to any of the nearest neighbor vacancies when these exist at that instant of time. If a movable lattice atom interacts with $m_{0}$ nearest neighbors $\left(m_{0}=\right.$ $\left.1, \ldots, m_{c}-1\right)$, then the activation free-energy barrier is $m_{0} \Delta>0$, and the hopping probability is proportional to $\exp \left(-m_{0} \Delta / k T\right)=p^{m_{0}}, p=e^{-\Delta / k T}<1$. In case the hop takes place, the atom occupies one of $\left(m_{c}-m_{0}+1\right)$ possible sites i.e. either one of the nearest $\left(m_{c}-m_{0}\right)$ 


\section{WILEY-VCH}

vacancies or stays at its original site. The probability of each state is proportional to temperature-dependent Boltzmann factors $\exp \left(m_{t}|\varepsilon| / k T\right)$ (normalized over all the $\left(m_{c}-\right.$ $m_{0}+1$ ) possible positions). The value $\varepsilon<0$ presents the free-energy of the pair binding, and $m_{t}$ is the number of nearest neighbors at the target sites. Note that a non-zero $m_{t} \neq 0$ $\left(m_{t}=1, \ldots, m_{c}-1\right)$ represents hopping, while $m_{t}=0$ signifies detachment when the bound atom rejoins the free diffuser gas.

Thus, in the presented mesoscale approach two thermodynamic parameters govern the nanowire shape evolution: $p$ and $\alpha$. The parameter $p$ is controlled by the activation freeenergy scale $\Delta$,

$$
p=e^{-\Delta / k T},
$$

and determines the surface diffusion coefficient; the second parameter,

$$
\alpha=|\varepsilon| / k T,
$$

corresponds to the free-energy scale, $\varepsilon$, that reflects local binding.

The results of previous numerical studies ${ }^{[30,40-46]}$ show that reference values of $\alpha_{0}$ and $p_{0}$, comparable to 1, may be used to describe typical nonequilibrium nanostructure morphologies of mesoscopic size in agreement with real experiments. When temperature, $T$, changes, the variations of the parameters $p$ and $\alpha$ satisfy the relation

$$
p=\left(p_{0}\right)^{\alpha / \alpha_{0}}
$$

Following prior experience with FCC nanocrystals ${ }^{[30,40-46]}$, we use the reference 'intermediate temperature' values for the MC-model rules: $\alpha_{0}=1$ and $p_{0}=0.7$, to establish the link between the values of $p$ and $\alpha$. 


\section{WILEY-VCH}

In the sequel, we present results of our numerical simulations for nanowire evolution in 'warm', $\alpha<\alpha_{0},(\alpha=0.9, p=0.725)$ and 'cold', $\alpha>\alpha_{0}$, regimes $(\alpha=1.2, p=0.652)$. As obtained by researchers ${ }^{[30,40-46]}$ this range of variation in parameter $\alpha$ well reflects the observed experimental patterns of the dynamic processes under investigation.

The physical characteristics of different metals with a face-centered structure (of distinguishable $\Delta / k T$ and $|\varepsilon| / k T$ ) vary and depend on a nanowire diameter. For instance, the typical temperatures to initiate break-up of gold, copper, and platinum nanowires are approximately $300^{\circ} \mathrm{C}, 400^{\circ} \mathrm{C}$, and $600^{\circ} \mathrm{C}$, respectively. In this article, the general patterns of nanowire disintegration, without reference to a specific metal, are examined.

A unit Monte-Carlo step is a random sweep through the system of free and bound atoms, so that diffuser-gas atoms move/hop once on average, and the lattice atoms have one attempt for changing their location according to aforementioned probabilistic rules.

At the initial state, the nanowire considered in our simulations contains around 200 thousand atoms (its diameter is approximately $d \sim 5 \mathrm{~nm}$, and its length $L$ up to $190 \mathrm{~nm}$ ). A small fraction of these atoms detaches from the nanowire surface for a short transient time to form a 'gas' in steady state with the nanocluster. The total number of atoms is, however, conserved because of the reflecting container walls.

Somehow, even the infinite nanowire breaks up into nanorods of different length. The ends of nanorods can affect the processes at their base parts. We therefore study nanowire dynamics for two initial configurations: (i) with 'free' ends, and (ii) with 'frozen' ends (Movies S3 and S4). In the second case, five additional atomic planes oriented perpendicular to the nanowire axis are inserted between each end and the end-wall of the container. These atomic layers of 


\section{WILEY-VCH}

moveless/frozen atoms slightly extend the nanowire and prevent the so-called 'end-effects' (see Supplementary Section S3 and Movies S1 and S2). To some extent, such an approach can be identified with periodic boundary conditions.

\section{Results}

\subsection{Morphology of neck formation}

In this section, we analyze the morphological properties of the formed necks which are common for nanowires not only of the FCC, but also of the BCC (Body-Centered Cubic) and diamond lattice structures (e.g., Germanium (Ge) and Silicon (Si)). Each case is characterized by a specific set of parameters of a Monte-Carlo (MC) model which, in turn, depend on the lattice coordination number. It is well-known that the global minimum of surface energy for the extended nanowire can only be achieved when it transforms into a single pseudo-spherical particle. However, the nanowire shape dynamics has to evolve in accordance with both the laws of motion and the laws of thermodynamics. The evolutionary 'trajectory' of the multiparticle system passes through many local minima and the disintegration of the nanowire into individual isomeric nanoparticles implies that the system has reached one of these local minima. Initial short-scale thermal perturbations, in the course of time, turn into long-wave radius modulations. The result of such 'sausage' instability (Movie S4) is the formation of well-defined necks (Fig. 2). The reduction in the surface energy of the system is achieved not only by reducing the total surface area (as happens in fluid streams) but also due to the heterogeneity/anisotropy of the density of this energy, $\sigma\left(\boldsymbol{e}_{n}\right)$, where $\boldsymbol{e}_{n}$ is the vector perpendicular to the surface $\left(\sigma\left(\boldsymbol{e}_{111}\right)<\sigma\left(\boldsymbol{e}_{100}\right)<\sigma\left(\boldsymbol{e}_{110}\right)^{[16]}\right)$. The type of the faces, as well as angles $\gamma, \beta_{1}, \beta_{2}$ (Fig. 2D), can be easily determined for a particular crystal lattice. Simple estimations, as shown below, allow us to attain a qualitative prediction of the dependence of the break-up parameter $\Lambda / r_{0}$ on the nanowire orientation. 


\section{WILEY-VCH}

It is well established that for large enough time-scales, an arbitrary nanocluster takes an equilibrium shape corresponding to a minimum of the total surface energy, which is close to the Wulff construction for the nanostructure under consideration ${ }^{[51-54]}$. For instance, the Wulff construction for gold is a truncated octahedron which is bounded by the (100)-, (111)-, and (110)-type planes, situated at approximately the same distance from the center ${ }^{[55]}$. We remind the reader that the Wulff construction represents a somewhat idealized form of a nanoparticle since its definition does not take into account kinetic processes associated with smoothing wedges and vertices.

The tendency to have an 'isomeric' surface is inherent not only to nanoparticles, but also to long nanowires, the surface of which has a predisposition to become bounded by certain crystalline facets ${ }^{[46]}$. After such nanowire reshaping, the facets that define the Wulff construction play the dominant role in the nanowire surface morphology. Regardless of the processes transpiring in the linear break-up stage, Wulff shape facets always appear at the final nonlinear stage. The results presented in (Fig. 2A) clearly demonstrate this property: the lateral surface of the [100]-oriented nanowire is bounded by four (100)-type facets and four (110)-type facets. During the nonlinear disintegration stage, eight faces of (111)-type form necks which eventually leads to the break-up $\left(\beta_{1}=\beta_{2} \approx 35.3^{\circ}, \gamma \approx 109.5^{\circ}\right)$. Therefore, for a nanowire with [100]-type orientation, fragments are symmetric well-faceted pre-drops before the breakdown (the necks seem to have axes of symmetry of the fourth-order). Generally, necks are bounded by facets, which intersect the nanowire axis at the small angles of $\beta_{1}$ and $\beta_{2}$, resulting in a reduction in the surface energy due to the decrease in the lateral surface area. 


\section{WILEY-VCH}
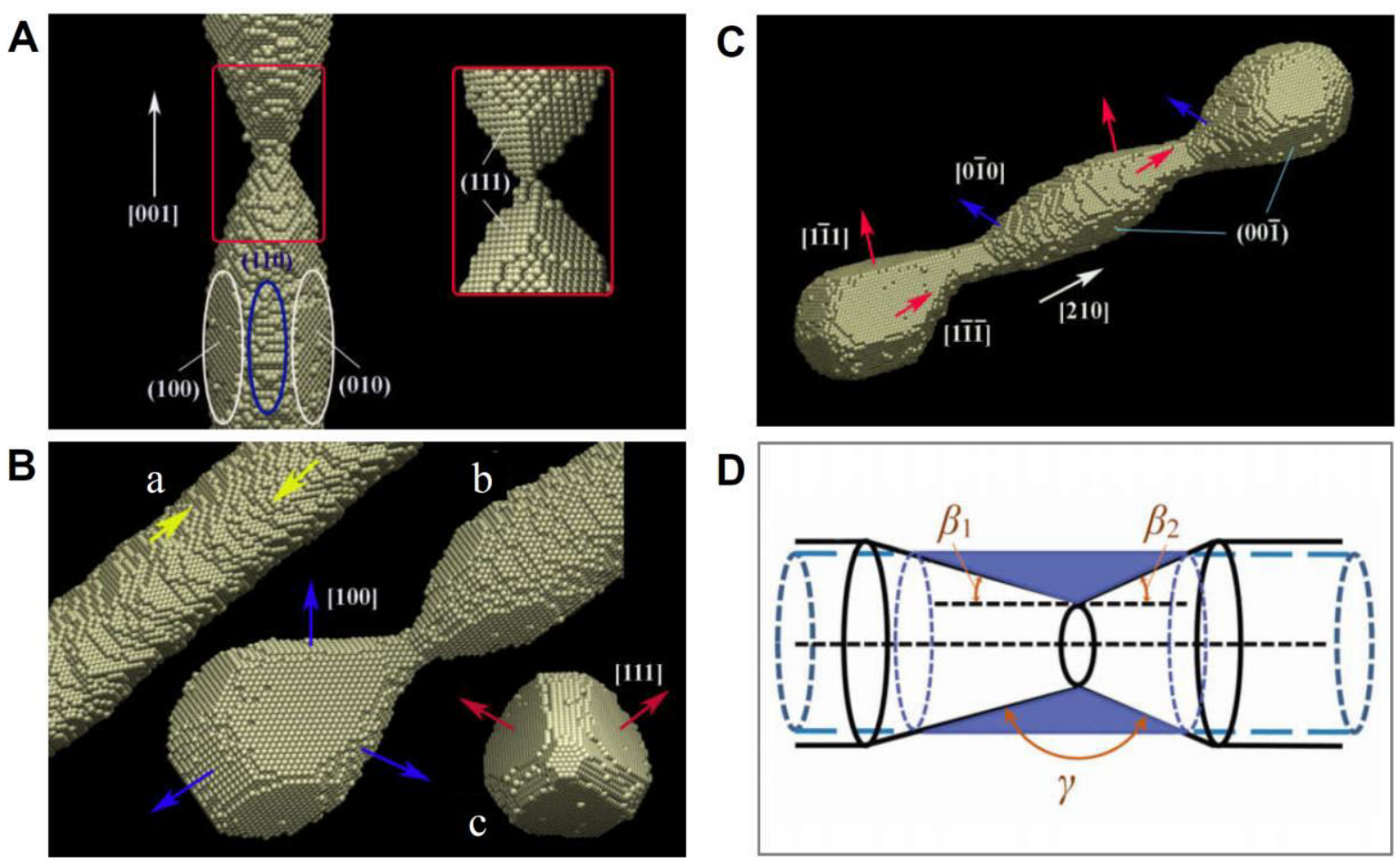

Fig. 2. Configurations of fragments in disintegrating nanowires with different axis orientations, $\boldsymbol{e}_{n}$, observed in the numerical simulations. (A) $\boldsymbol{e}_{n}=[100]$; structure of the lateral surface of the pre-drops (on the left) and the necks (on the right) exactly at the break-up time. (B) $\boldsymbol{e}_{n}=$ [111]; subpart (a) displays the structure of the lateral surface of the nanowire prior to the occurrence of necking; subparts (b) and (c) show the shapes of the end of the extended nanorod just before and immediately after its break-up. Red and blue normal vectors designate types of bounding facets. (C) $\boldsymbol{e}_{n}=$ [210]; an example of a relatively short nanorod, formed during the break-up of a longer nanowire. (D) An idealized geometric model of the formed bottleneck and its associated parameters. Blue dashed lines represent the initial configuration of a generic nanorod. 


\section{WILEY-VCH}

Let us conduct a qualitative analysis of surface dynamics for a nanowire with [111]-type orientation. For a given volume, the smallest surface energy is associated with the nanowire bounded by six (110)-planes. The next step in the energy reduction is the transformation into six longitudinal strips, with the step-like structure (subpart (a) in Fig. 2B) consisting of the fragments of (111)-type planes. The form of necks (subparts (b) and (c) in Fig. 3B) can be easily predicted. They are formed by six planes, three planes with (111)-type orientation and three with (100)-type that intersect the nanowire axis at approximate angles of $\sim 19.5^{\circ}$ and $\sim 35.3^{\circ}$, respectively (with the angle between opposite faces being $\gamma \approx 125.3^{\circ}$ ). Thus, the necks look like a symmetrical construction with an axis of symmetry of the third-order.

It is relatively easy to establish that the side surface of the cylindrical nanowire with [110]type orientation will eventually become symmetrically bounded by four planes of $(-11 \pm 1)$ and $(1-1 \pm 1)$-types and two planes of $(00 \pm 1)$-type. The bottlenecks of second-order symmetry can be formed only by the planes of the same type $((11 \pm 1),(-1-1 \pm$ $1),( \pm 100)$, or $(0 \pm 10))$, but these planes intersect the nanowire axis at very large angles $\left(\sim 54^{\circ}\right.$ and $\left.45^{\circ}\right)$. Since the four side planes of (111)-type have the lowest surface energy density in the FCC-lattice, the formation of periodic necks becomes energetically disadvantageous in the case of insufficient reduction (due to large angles $\beta_{1,2}$ ) in the total surface area. Therefore, the [110]-type orientation is very stable with respect to the break-up into fragments, a fact which was indicated in previous works ${ }^{[27,46]}$. Furthermore, the 'endeffect' (the specific break-up mechanism, which is discussed below) does not hold for this structure either, due to the high surface diffusion rate of atoms deposited on nanowire (111)facets (because of low binding energy). Analogous situations can be realized in nanorods with other types of crystalline structure. 


\section{WILEY-VCH}

For the [321]-, [211]-, [221]-, [210]- and [320]-type orientations, the nanowire axis no longer represents the axis of symmetry during the necking process. In such cases, several features of the break-up, which cannot be explained using the existing model ${ }^{[24]}$, can be observed. The discussion of the results of numerical experiments for the non-symmetrical type orientations (listed above) will be presented below. For now, we only note the similarity between the [321] and [211] orientations: in both cases, the initially cylindrical wire becomes faceted, by only two (1-1-1) and (-111) planes bounding it (as opposed to the [110] orientation with four such facets).

It is necessary, at this juncture, to discuss the [210] orientation since it shows a distinct asymmetry of the forming neck with respect to rotations around the axis of the nanowire. After slight flattening by $(00 \pm 1)$ planes, eight facets of (111)-type (which have the lowest surface energy density) intersect the nanowire axis at acute angles (see Fig. $2 \mathrm{C}$ where the presented configuration is symmetric under rotation by angle $\pi$ around the axis perpendicular to the nanowire). Left/right parts of the neck are formed by a pair of adjacent $(1-1 \pm 1)$ planes with an approximately $15^{\circ}$ tilt angle with respect to the nanowire axis, the $(0 \pm 10)$ planes (tilt angle $\sim 26.6^{\circ}$ ) and step-like slopes with an average inclination angle $\sim 35^{\circ}$. Comparing the obtained values with the results of the same analyses but on [100]- and [111]type orientations leads to the following conjecture: the time required for the nanowire breakup, and the value of the parameter $\Lambda / r_{0}$ will significantly increase for [210]-type orientations. Indeed, the case when the angles $\beta_{1}$, and $\beta_{2}$ are equal for any value of angle $\gamma$ corresponds to the minimum length of the neck and the minimum volume of matter (marked by blue in Fig. 2C) which has to be displaced during the development of instability. Once the symmetry is broken, both these parameters (the length of the neck and the displaced volume of matter) increase. Additionally, angle $\gamma$ has the largest average value for the [210]-type orientation. 


\section{WILEY-VCH}

Therefore, on the basis of the estimations above, the result obtained in numerical simulations, $\Lambda / r_{0} \sim 13$, is predictable.

\subsection{Linear stage of break-up in nanowires}

Surface evolution in a long nanowire strongly depends on its orientation, precisely at the nonlinear stage of its necking, when the difference in the morphology of the forming necks is fully manifested (see above). Scenarios of the linear stage do have individual features for different nanowire orientations. In particular, the effects of restructuring the nanowire surface as a result of arising thermal roughening ${ }^{[37,38,39]}$ is sensitive to the types of facets that form its lateral surface. However, in general, the physical mechanisms that determine the $\lambda_{\max }(t)$ dynamics are identical; here we consider the surface processes in detail only for the case of [100]-nanowire (Fig. 3). 


\section{WILEY-VCH}

A

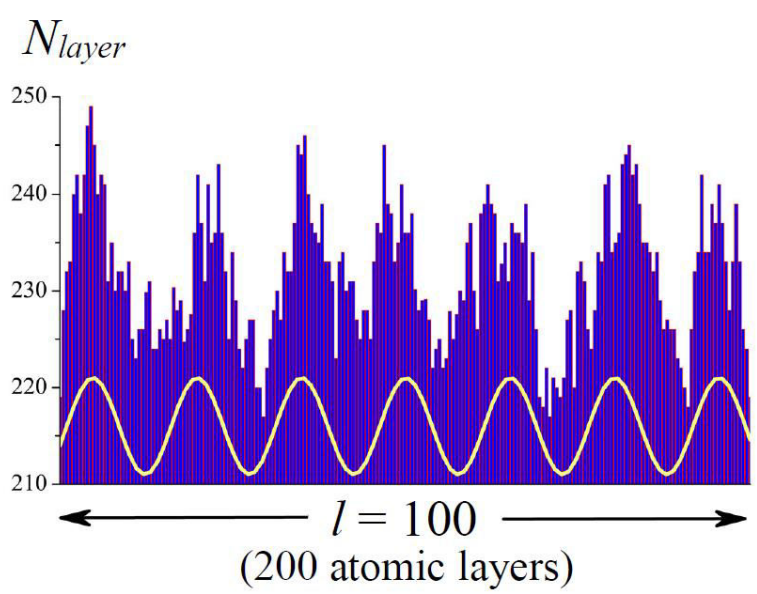

B

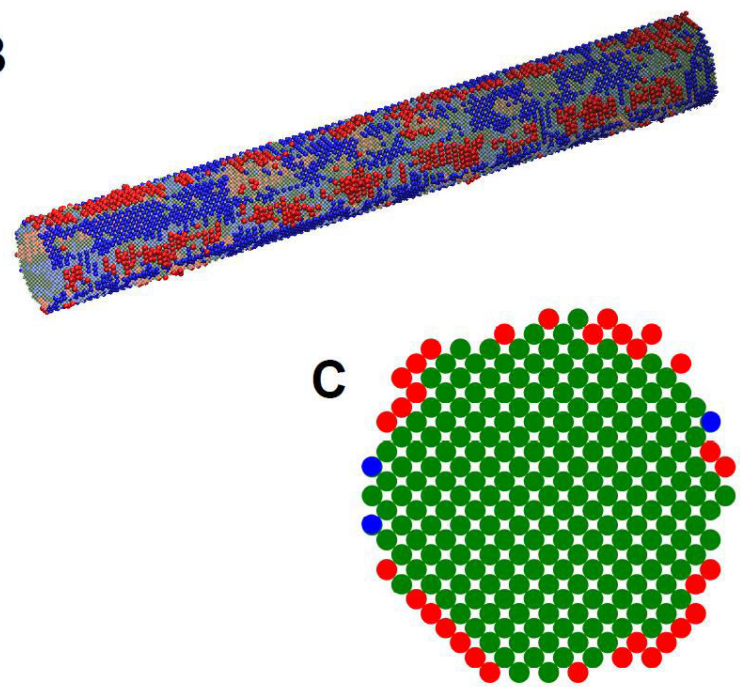

D
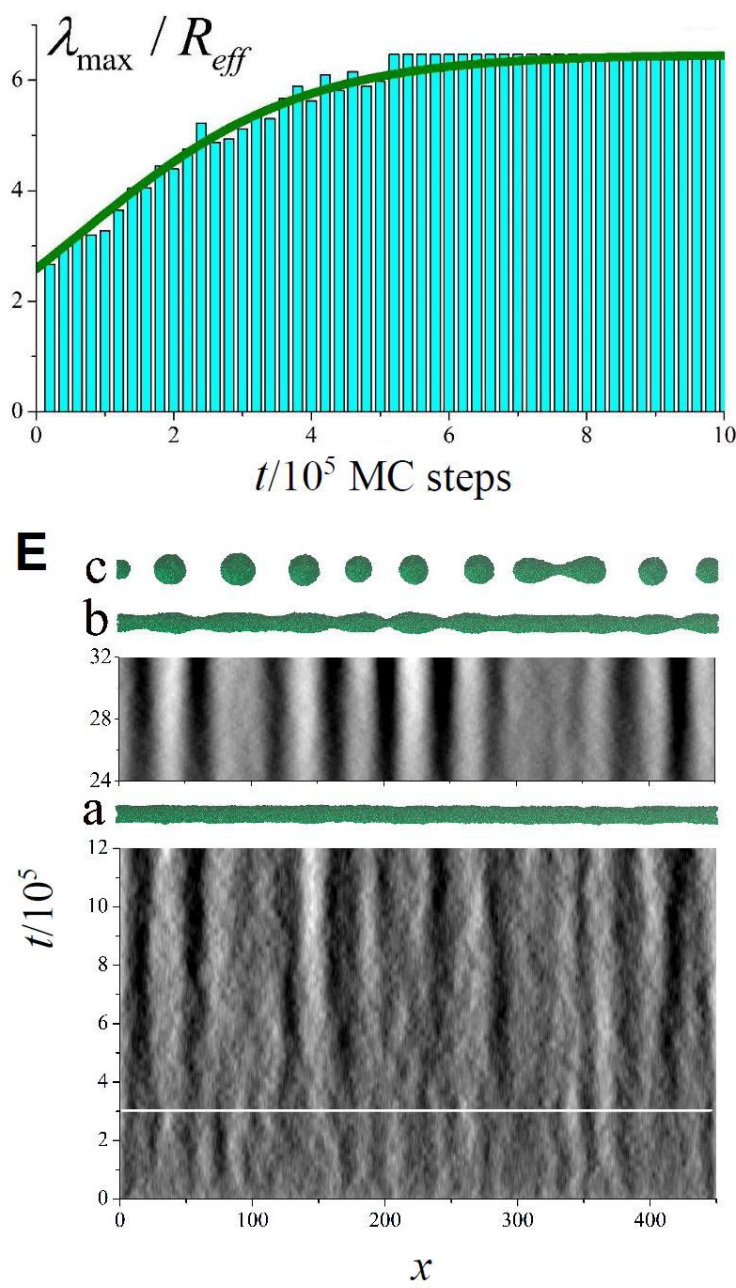

Fig. 3. Dynamics of the nanowire surface morphology. ([100]-orientation, 'warm' regime, $L=450$, and $d=12$.) (A) A fragment of the distribution of the number of atoms, $N_{\text {layer }}(x, t)$, in atomic layers (100) along the nanowire at time, $t=2 \times 10^{4} \mathrm{MC}$ steps. Yellow curve in this figure depicts the harmonic with $\lambda=\lambda_{\max }(t)$, which has maximum amplitude in the Fourier transform of the function $N_{\text {layer }}(x, t)$. (B) The shape of the nanowire fragment presented in Fig. 3A compared to its initial shape. Glass-like spheres present the 'common' atoms for both superposed snapshots at MC step instants $t=0$ and $t=2 \times 10^{4}$. The blue atoms belong only to the initial nanowire shape. Until $t=2 \times 10^{4}$, these blue spheres indicate the creation of near-surface vacancies. The nanoclusters of red atoms occupy the vacancies around the initial nanowire and cause the variation in the spatial variation manifested as $N_{\text {layer }}(x)$ (see Fig. 3A). (C) The distribution of atoms in two (100)-layers with a minimum and maximum number of atoms (see layers (a) and (b) marked in Fig. 3A). Olive circles represent the common atoms that belong to both (a) and (b) layers; the layer (a) consists of both the olive and blue atoms; the layer (b) is represented by both the olive and red atoms. (D) The dependence of $\lambda_{\max }(t)$ at the 'linear' disintegration stage, $2 R_{\text {eff }} \approx 11.6$ (4.7 $\mathrm{nm}$ for Au). Olive curve represents the approximating function, $\lambda_{\max } / R_{\text {eff }} \approx 6.5 \times\left[1+1.5 \times \exp \left(-\frac{t}{1.6 \times 10^{5}}\right)\right]^{-1}$, for $t \lesssim 10^{6}$. Grayscale map of $N_{\text {layer }}(x, t)$ spatial-time dynamics. Subparts (a), (b), and (c) depict the shape of the nanowire at $t=\{12,32,59\}\left(\times 10^{5}\right)$, respectively. 


\section{WILEY-VCH}

The periodic spatial modulations of the number of atoms in the planes (100) (Fig. 3A) arise due to the formation of a fairly ordered structure of short-scale surface clusters. The clusters themselves are the result of thermal fluctuations/roughening that transfer atoms from the (100)-facets to the (110)-facets, where these atoms are trapped by the near-surface vacancies with higher binding energy (see Fig. 3B and Fig. 3C (red atoms)). In Fig. 3D, averaged over twenty realizations, the temporal evolution of $\lambda_{\max }(t) / R_{\text {eff }}$ is shown. Here, $\lambda_{\max }(t)$ is the maximum amplitude in the Fourier transform of the number of atoms, $N_{\text {layer }}(x, t)$, in the (100) planes; $R_{e f f}$ is the nanowire radius when a steady equilibrium state between the nanorod and the vapor of free atoms, which fill the surrounding container bounded by the reflecting walls, is established for a time much shorter than the time needed for the break-up to occur:

$$
R_{e f f} \approx r_{0} \sqrt{N_{t} / N_{0}}
$$

where $r_{0}$ is the initial radius of the nanowire, $N_{0}$ and $N_{t}$ are the initial number of atoms and their number in the nanorod in the quasi-equilibrium state, respectively.

The transformation of short-scale surface perturbations into long-scale ones may be tracked in Fig. 3E, where a grayscale-map of the space-time distribution, $N_{\text {layer }}(x, t)$, obtained in a numerical simulation, is presented. This transition is clearly visible above the white horizontal line (corresponding to $t>3 \times 10^{5}$ in Fig. 3E). It is observed that at the time interval, $t \sim(3-4) \times 10^{5}$, the average number of bonds per atom, $\left\langle n_{b}(t)\right\rangle$, reaches a minimum of $\sim 11.193$, as compared to the initial value $\left\langle n_{b}(0)\right\rangle \sim 11.216$, which implies that termal roughening has finished restructuring the nanowire surface (See Supplementary S1).

At first sight, the appearance of the nanowire configuration at $t=12 \times 10^{5}$, which is shown in subpart (a) of Fig. 3E, corresponds, to a greater extent, to the chaotic fluctuations of the 
surface. However, in reality, there is the explicit structure in the distribution $N_{\text {layer }}(x, t=$ $12 \times 10^{5}$ ) clearly observed in the grayscale-map.

The dynamical parameter $\lambda_{\max }(t) / R_{e f f} \sim 6.5$ is almost unchangeable for a fairly long time. This value is slightly higher than the critical value, $2 \pi$, which is the necessary threshold for the further development of the necks in time but is not sufficient for a break-up. On the one hand, according to the results obtained in the previous section, the necking slopes should be oriented (as claimed by the crystal structure) at fairly small angles with respect to the nanowire axis, which provides a greater reduction in the total surface area, when these necks experience noticeable narrowing. On the other hand, the short gaps between them is in conflict with the necessity to have enough volume to accommodate the substance that is removed from the zones of narrowing. Thus, the jet breaks can be realized only as a result of the competitive 'absorption' of the necks by each other (once again, the neck slopes cannot be formed in an arbitrary manner and should be dominantly constructed of the 'main' facets forming the Wulff shape). It is this process, that occurs at the nonlinear stage of decay and is highly random - See subparts (b) and (c) of Fig. 3E. Besides, one can see a long-living 2body dumbbell with further unpredictable evolution: either merging in the future or breaking up into two nanoparticles (Movie S5). Both future scenarios are conceivable, if the configuration depicted in subpart (c) of Fig. 3E is treated as the initial state (base configuration) for several random numerical experiments (Supplementary Section S4).

As a final remark on this section, it must be mentioned that the linear break-up stages are similar for the set of nanowire-orientations under consideration as long as the dynamical parameter, $\lambda_{\max }(t) / R_{e f f}$, is low $\left(\lambda_{\max }(t) / R_{e f f} \lesssim 2 \pi\right)$. The nonlinear stage (stage of 'competition' among neckings) is strongly specific for 'nonsymmetrical' orientations. The 
break-up dynamics of [321], [211], [210], [221] and [320] nanowires, as typical representatives of this set, is discussed in the sequel.

\subsection{Nonlinear stage of break-up in nanowires}

The break-up of nanowires with orientations (100) and (111) is discussed in detail in Supplementary S1. In these cases, the axis of the wire is the axis of rotational symmetry of order 4 or 3 , and the sausage-like shape perturbations along this axis resemble those of a disintegrating liquid jet. Besides, existing analytical models of instability ${ }^{[27,28]}$ and physical experiments give similar results for the break-up parameter $\Lambda / R_{e f f}$, despite the nontrivial evolution path from the linear stage to the break-up stage with a number of random breakdowns and the possible formation of long-lived 2-3-body dumbbells $\left(\Lambda / R_{\text {eff }} \approx 9 \pm 0.3\right.$, Supplementary Section S1).

This section is devoted to the investigation of the self-consistent effects of anisotropy of surface energy and asymmetry of the necks. In the sequel, we shall discuss the break-up features of nanowires with [321], [210], [211], [221] and [320]-type orientations. In all these cases, the presence of the crystal structure of the nanowire is clearly manifested visually as well as quantitatively. A set of 'trajectories' of the system with a decreasing value of surface energy should be associated not only with the reduction of surface area but also with different energy densities at distinct regions of the forming necks. In the cases under consideration, the crystallographic faces that dominate in the formation of necks intersect the wire axis at smaller angles than in the cases of [100] and [111]-type orientations. We recall that it follows from simple geometric relations (see the first section of the Results) that even if only one angle $\beta_{1,2}$ (Fig. 2D) is small, the mass of the substance extracted from the neck area significantly increases and the neck consequently extends. As a result, the value of the breakup parameter $\Lambda / R_{\text {eff }}$ exceeds, by $20-40 \%$, unity, which was obtained by the model based on 


\section{WILEY-VCH}

the assumption of the isotropy of surface energy ${ }^{[28]}$. Consequently, the break-up time under the same initial conditions increases. Furthermore, at the linear break-up stage, $w$-like fractures of the surface can occur the origin of which is the existence and manifestation of the nanowire's rotational asymmetric crystalline structure.

Earlier in this study, we discussed only the anisotropy of parameters which are directly measured during experiments. However, there are several factors, which are hidden from an observer and related to the structure of lateral surfaces of the nanowire. This structure determines the anisotropy of the surface diffusion coefficient, which is manifested in the following effects. Generally, the nanowire splits into a series of extended fragments at the initial stage. The further evolution of fragments, to a great extent, depends on the role of endeffects in the subsequent break-up into droplets. In turn, the role of end-effects is smaller if intensive surface diffusion suppresses the formation of bulbous structures at the nanowire ends. A demonstration of these processes is presented in Figs. 4 and 6.

Based on the data presented in Fig. 4, the break-up parameter varies in the range $\Lambda / R_{e f f} \approx$ 11 - 13. In our numerical simulations with [321]-orientation, five drops were formed in one numerical experiment out of six. This is unusual as normally four drops form, but we note that even in such rare cases for a nanowire with $L=350$ and $R_{\text {eff }}=6.35$, the break-up parameter is around $\Lambda / R_{e f f} \approx 11$. The effect of the interference of short-wave surface perturbations near the left end of the nanowire with the waves generated by the end-effect is demonstrated in the subparts (a) and (b) of Fig. 4C. One can see the protracted dynamics of the extended nanorod, monotonically decreasing in length, as shown in the subparts (d) to (g) of Fig. 4B. The reason for this is that in the case of [321]-type orientation, two (111)-type faces flatten the initial cylindrical shape of the wire. As mentioned earlier, the rate of surface diffusion on 


\section{WILEY-VCH}

these faces is high, which suppresses end-effects followed by the separation of drops due to the intense outflow of atoms into the middle part of the wire.

In the nanowires with [210]-type orientation the averaged value of the break-up parameter gives $\Lambda / R_{\text {eff }} \approx 13$. The examples of the evolution of such nanowires with 'free' and 'frozen' ends are presented in Fig.5. In the section titled 'the morphology of neck formation', when discussing the properties of the crystallographic planes that form the necks, we indicated the possibility of the formation of long-wave perturbations already at the linear break-up stage. This assumption is confirmed by the data presented in Fig.5B (see the configuration (a) and the corresponding distribution, $\widehat{N}_{\text {layer }}(x, t)$, where $\left.\lambda_{\max } / R_{\text {eff }} \approx 12\right)$. In should be noted that four facets of (111)-type are oriented at a small angle $\left(15^{0}\right)$ with respect to the nanowire axis and their fragments form the step-like lateral surface of the wire (Fig. 5B, the inset on the left), with the high density of the corner vacancies that significantly reduces the rate of surface diffusion. This is the reason that the end-effect mechanism, causing the separation of drops from the ends of elongated nanorods (see Supplementary S3), is more effective in [210]nanowires (Fig. S4A and B) than in nanorods with (321)-type orientation (subparts (d) to (g) of Fig. 4B). 


\section{WILEY-VCH}
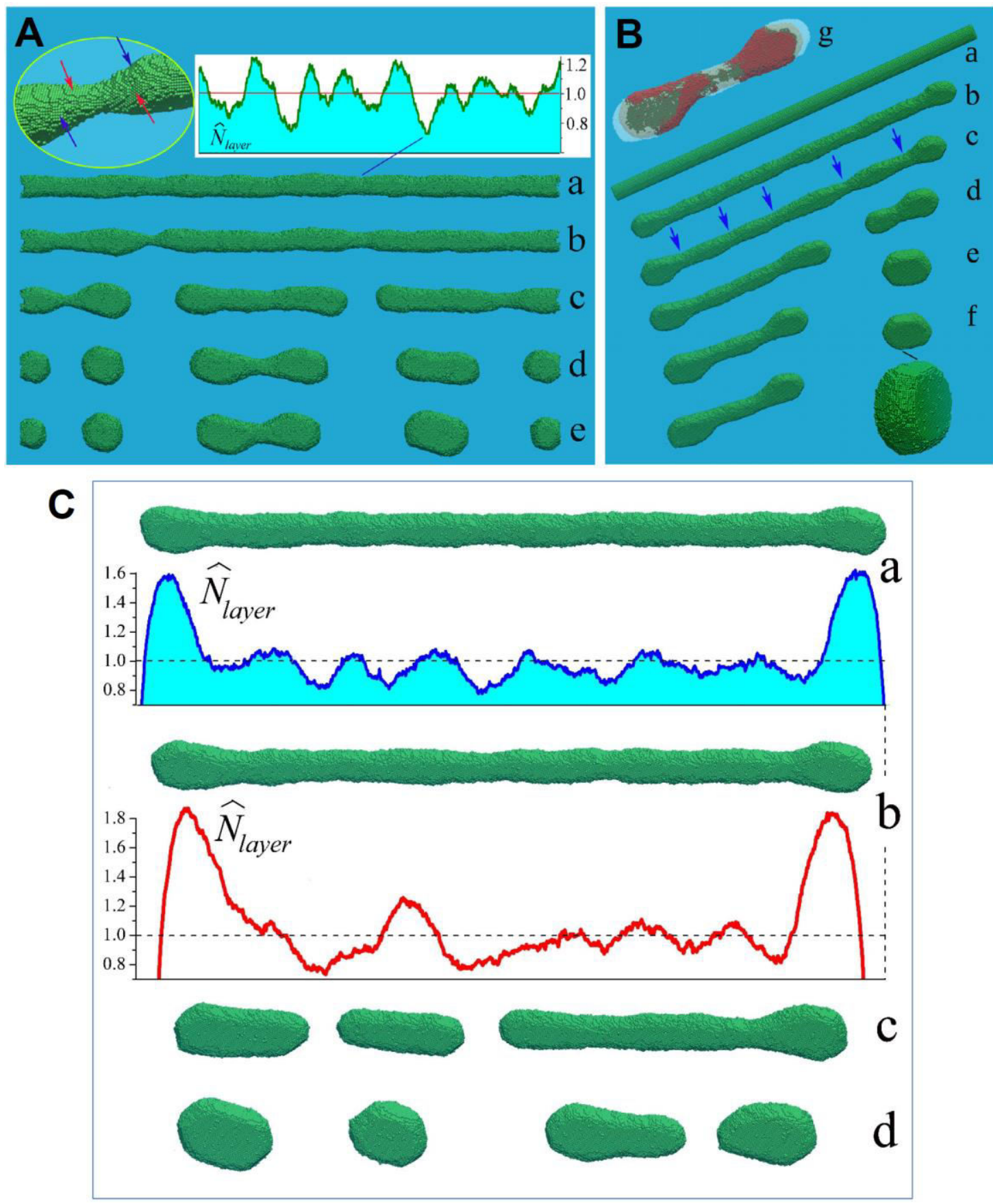

Fig. 4. Disintegration process of a [321]-orientation nanowire with 'frozen' and 'free' ends. Results for the warm regime: $\alpha=0.9, p=0.725, L=350$, and $d=13$. (A) Frozen ends; configurations (a) to (e) show the system at $t=3,5,7,10,13 \times 10^{6}$, respectively. The inset on the left shows the shape of the neck in the configuration (b), red/blue arrows indicate the fragments of (111) and (100) planes, respectively. The inset on the right shows the distribution of the number of atoms in atomic layers perpendicular to the axis of the nanowire, where $\widehat{N}_{\text {layer }}(x, t)=N_{\text {layer }}(x, t) /$ $N_{\text {layer }}(t=0)$. (B and $\left.\mathbf{C}\right)$ Two random MC simulations of the nanowire with free ends. (B) Subparts (a) to (f) correspond tot $=0,3,6,10,15,22 \times 10^{6}$, respectively. Arrows indicate the locations of the necks. Subpart ( $\mathrm{g}$ ) illustrates the combined shapes of the nanorod at $\mathrm{t}=22 \times 10^{6}$ and $\mathrm{t}=25.2 \times$ $10^{6}$; olive atoms are common to both moments; glass-like atoms and red atoms represent changes in the form of the nanorod with time. (C) Subparts (a) to (d) correspond to $t=2.4,4.0,8.0,14.6 \times 10^{6}$, respectively. At the first two instants (i.e. a and b) the distributions of the number, $\widehat{N}_{\text {layer }}$, of atoms in the (321)-layers along the nanowire are shown. In every case $N_{0} \sim 185.2$ thousand atoms, and $N_{t} \sim 176$ thousands. 


\section{WILEY-VCH}
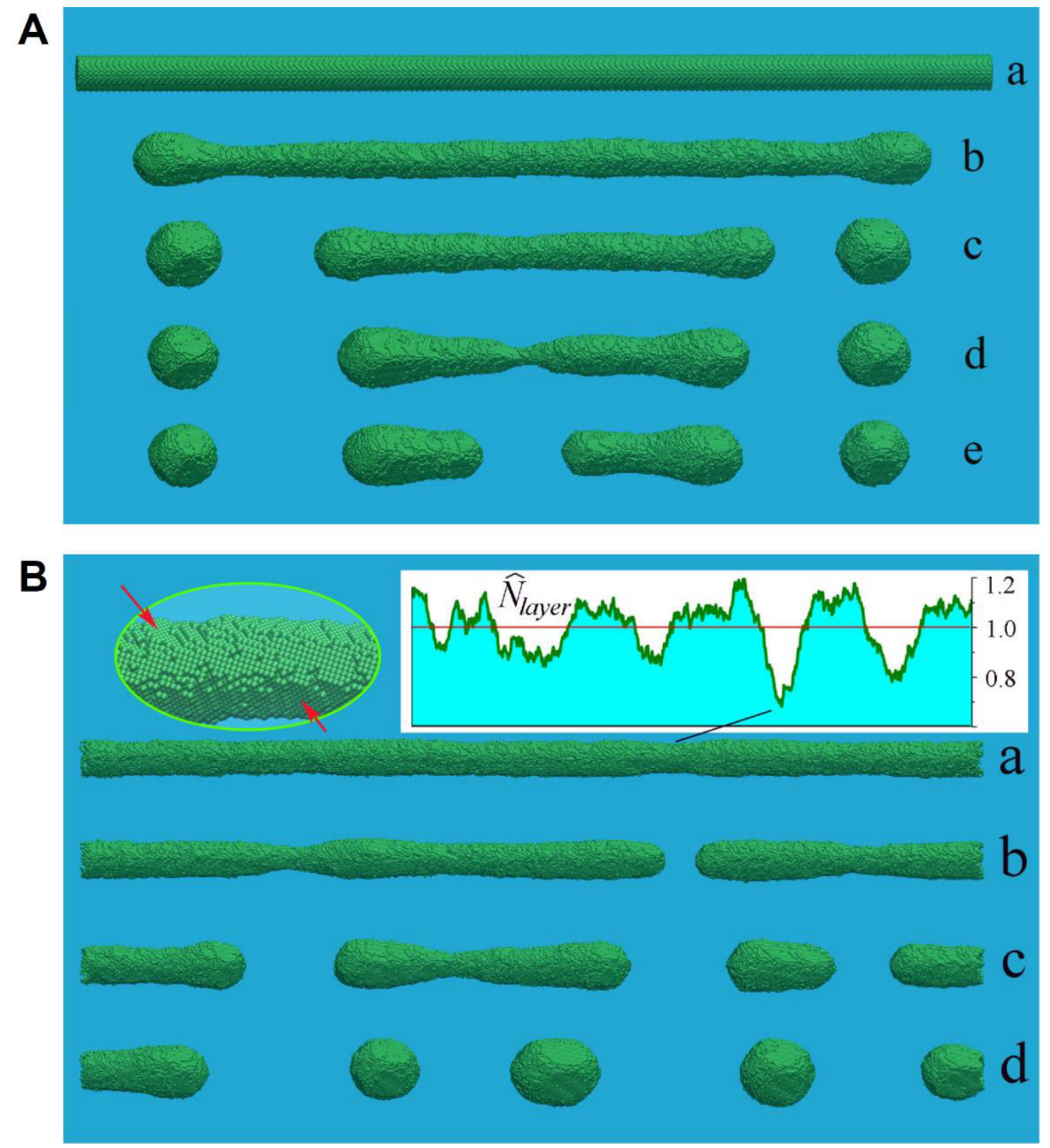

Fig. 5. Break-up of nanowires with orientation [210]. Results for the (A) 'frozen' and (B) 'free' ends (warm regime: $\alpha=0.9, p=0.725, L=350 a$, and $d=13 a$ ). In the subparts (a) to (e) of part A: $t=0,4,8,11,12.2 \times 10^{6}, N_{0} \sim 183.5$ thousand atoms and $N_{t} \sim 173.4$ thousands. In the subparts (a) to (d) of part B: $t=3,6,8,12 \times 10^{6}$. The insets show the structure of the lateral surface and distribution of the number of atoms in the (210)-type layers at $t=3 \times 10^{6}$. 


\section{WILEY-VCH}

The [211]-type orientation also possesses several unique properties. Two (111)-type facets flatten the initial cylindrical form of the nanowire. As a result, the necks are formed at the top and bottom sides of the nanowire (Fig. 6), i.e. on the lateral sides of the quasi-twodimensional configuration. We note that the left and right neck slopes are built in the same way. Each slope consists of a pair of the (111)- and (100)-type facets. The fact that the angles of inclination with respect to the axis of the nanowire are small $\left(\sim 28^{\circ}\right.$ and $\sim 13.6^{\circ}$, respectively) means that a large mass should be removed from the neck region and transferred along the nanowire. Local minima of the surface energy in such a system can be achieved after the shift of constrictions at the top with respect to the constrictions at the bottom. This results in the formation of regions with snake-like ( $w$-like) shape (see e.g. in Fig. 6 the zones enclosed by a blue ellipse). The high rate of surface diffusion on the bounding planes suppresses the break-up of 2-3-body dumbbells even if they have small sizes of necks (see the 2-body dumbbells, numbered as 1 and 2, in Fig. 6(c)). It can be seen that the initial short-wave perturbations do not transform into a series of droplets. As a result, the break-up parameter has a significantly higher value than it was predicted by the linear theory: $\Lambda / R_{e f f} \approx 11$. 


\section{WILEY-VCH}

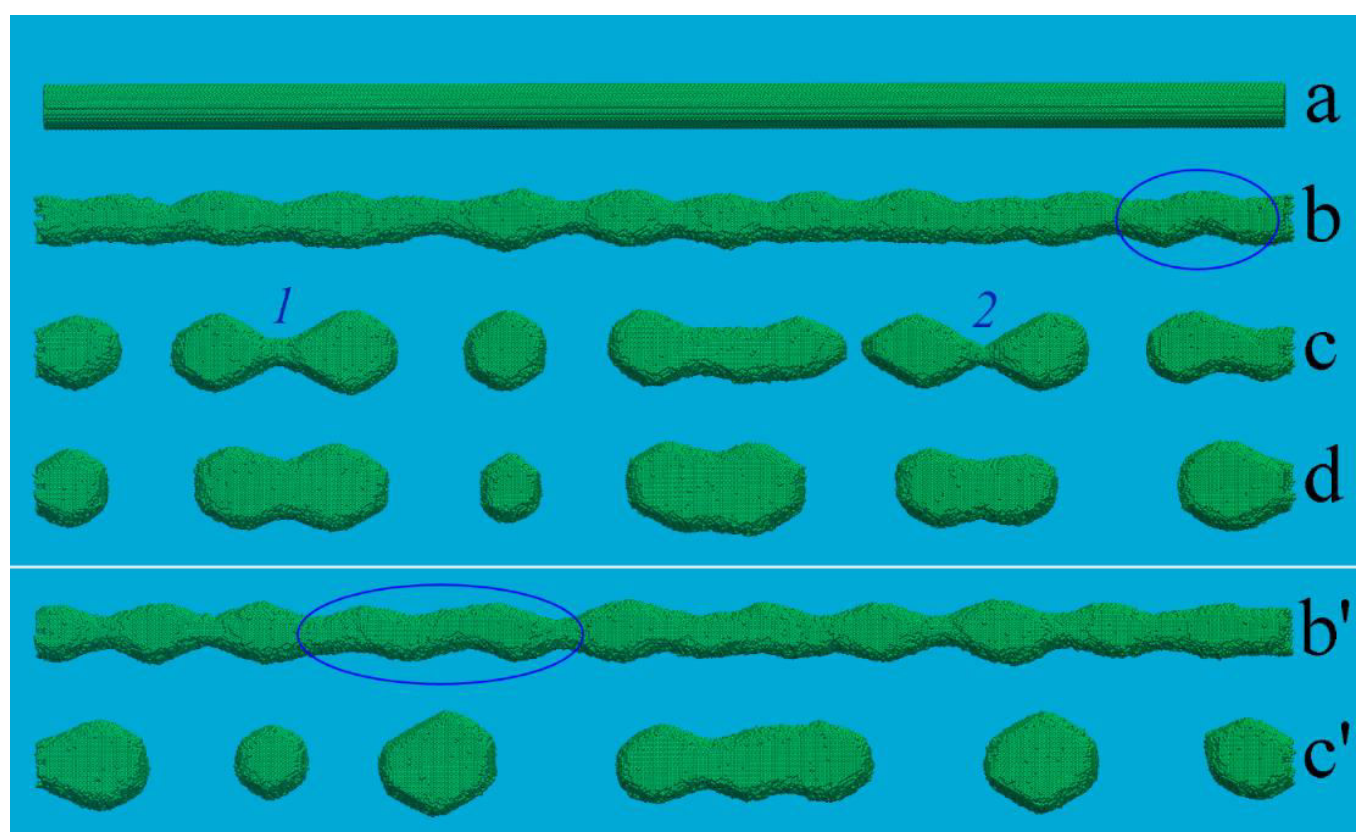

Fig. 6. Disintegration of a [211] nanowire at the warm regime. Results for $\alpha=0.9, p=0.725$, $L=360$, and $d=12.3$. The initial number of atoms $N_{0}=169800$; the number of particles in an equilibrium state $N_{t} \approx 167800$. Subpart (a) illustrates the initial shape, while subparts (b), (c), and (d) show snapshots taken at $t=3.75,10.2$, and $18.0 \times 10^{6}$, respectively. Subparts (b') and (c') depict the results of another random simulation of the process at $t=5 \times 10^{6}$ and $t=18 \times 10^{6}$, respectively. Blue ellipses mark w-like fractures of the nanorod. 


\section{WILEY-VCH}

In the conclusion of this section we examine nanowires with [221]- and [320]-type orientations. Both of these orientations (as well as the [210] orientation) are similar in that at least four planes of (111)-type, that have the minimal surface energy density, take part in the formation of the neck regions. The angles of inclination of these planes with respect to the axis of the nanowire are smaller than for the [111] orientation, which is discussed in detail in Supplementary S1. For this reason, for all three asymmetrical orientations ([221], [320] and [210]), the break-up parameter $\Lambda / R_{\text {eff }}$ must be greater than $9\left(\Lambda / R_{\text {eff }} \sim 13\right.$ for [210] orientation, see above). However, the neck regions are formed not only by (111) planes. As a result, the break-up parameter for similar, at first sight, main factors of the break-up can vary in a wide range: $\Lambda / R_{e f f} \sim 11$ for [221] and $\Lambda / R_{e f f} \sim 16$ for [320] orientations, as it is shown in the sequel.

Recall that in case of the [111]-orientation, axisymmetric necks (Fig. 2B) are formed by six (111)-type (intersecting the nanowire axis at the same angle $\beta_{111} \approx 19.5^{\circ}$ ) and six (100)-type planes $\left(\beta_{100} \approx 35.3^{\circ}\right)$ and the corresponding break-up parameter is $\Lambda / R_{\text {eff }} \approx 9$ (See S1). In the [221]-type orientation, the symmetry in the neck region is broken. Four out of six (111)type planes intersect the nanowire axis at a smaller angle $\beta_{-11 \pm 1}=\beta_{1-1 \pm 1} \approx 11.1^{\circ}<19.5^{\circ}$, while for the other two, the angle is approximately $\beta_{11-1}=\beta_{-1-11} \approx 35.3^{\circ}>19.5^{\circ}$. A similar "skewness" also takes place for (100)-type planes where: $\beta_{0 \pm 10}=\beta_{ \pm 100} \approx$ $41.8^{\circ}>35.3^{\circ}$, and $\beta_{00 \pm 1} \approx 19.5^{\circ}<35.3^{\circ}$.

The results, shown in Fig. 7, clearly demonstrate the nonlinear effect of absorption of shortwavelength surface perturbations by each other (see the olive/red ellipse in Fig. 7A). The assumed number of droplets, $N_{\text {drop }}$, at the last stage of break-up could be predicted by the ratio of the length of the nanowire, $L$, to the harmonic wavelength, $\lambda_{\max }$, with the maximum amplitude in the Fourier transform of $N_{\text {layer }}(l, t)$ in space: $N_{\text {drop }} \approx L / \lambda_{\text {max }}$. However, such an estimation at the linear stage $\left(L / \lambda_{\max } \approx 10.7\right.$ at $t=t_{a}$ (Fig. 7A)) is totally inconsistent with the obtained results, $N_{\text {drop }} \approx 6$ (see the chains of nanodrops in the subparts (c) and (c') of Fig. 7A. Symmetry breaking in the neck region is especially noticeable in the configurations (a) and (c) of Fig. 7B and the rather strong anisotropy of the surface energy causes an increase in the break-up parameter, $\Lambda / R_{e f f} \approx 10.8$ as compared with the case of isotropic surface energy. 


\section{WILEY-VCH}

An example of a record breakup parameter is shown in Fig. 7C. The axis of the [320] oriented nanowire deviates by approximately $11.3^{\circ}$ from the stable orientation [111]. At the initial stages of transformation, the round wire is slightly flattened by the (100)-type faces (See the subpart (a) of Fig. 7C), and the four (111)-type planes, crossing the wire axis at an acute angle of $\approx 9.2^{\circ}$, form its scaled lateral surface. Naturally, under such conditions, rather long-wave perturbations must dominate at the nonlinear decay stage. According to the subpart (b) of Fig. 7C, the estimation of the value $\lambda_{\max } / R_{\text {eff }}$ is approximately 15 . The final stage of the nanowire disintegration is presented in Fig.7C(c). The averaged value $\Lambda / R_{\text {eff }} \approx 16$. As a final statement, we note that such a high value of the break-up parameter cannot be obtained by using existing models ${ }^{[20,24]}$. Moreover, the ratio of the average gap between neighboring nanodrops to their average size/diameter (when they become isomeric/ even-proportioned with time) is approximately 2.6 which is in agreement with the experimental results ${ }^{[25,27]}$.

For all the orientations considered, we have studied the role of the exchange of atoms between the nanowire surface and the surrounding vapor of free atoms, by artificially changing the dynamics rules in our MC model. If an atom were given the chance to break away from the surface of the nanowire according to the original algorithm, then such an attempt was canceled. This adjustment makes it possible to compare the contribution to the break-up dynamics of surface diffusion of atoms and their transfer in the near-surface layer for different cases. It is physically clear that the absence of vapor may reduce the effect of thermal roughening and delay the break-up time. Strong anisotropy was also manifested in these numerical experiments. Symmetric [111] -orientation is the most sensitive to the absence of surface-vapor exchange (the wavelength of the surface perturbations increases significantly and the breakup time increases by 5-7 times - See Supplementary S1). Asymmetrical orientations react weaker to the changes in the dynamic rules (breakup is delayed by 2-3 times), while with a symmetric [100] orientation, the dynamics of the nanowire remains almost unchanged (S1). 


\section{WILEY-VCH}
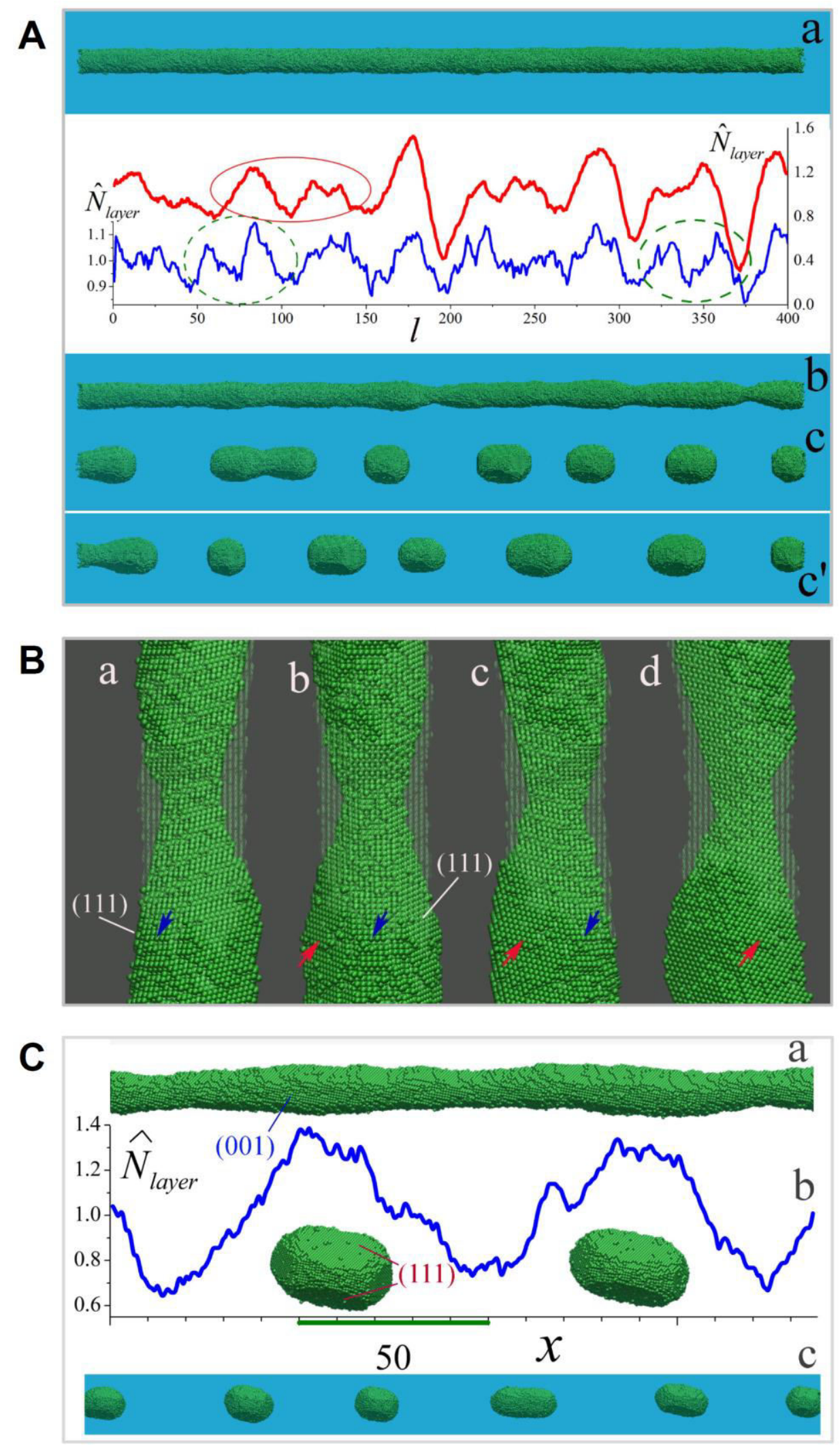

Fig. 7. Break-up dynamics of nanowires with different orientations in the 'warm' regime. Warm regime; $L=400, d=12.5\left(N_{0}=199 \times 10^{3}, N_{t}=192.6 \times 10^{3}\right.$, and $\left.R_{e f f} \approx 6.15\right)$. (A and B) The [221]-type orientation; $L=400$ and $d=12.5\left(N_{0}=199 \times 10^{3}, N_{t}=192.6 \times 10^{3}\right.$, and $R_{e f f} \approx$ 6.15). (A) The nanowire shape at the time (a) $t_{a}=10^{6}$, (b) $t_{b}=3.3 \times 10^{6}$ and (c) $t_{c}=8.7 \times 10^{6}$. 


\section{WILEY-VCH}

Snapshot $\left(c^{\prime}\right)$ shows the result of another random MC simulation of the break-up process for the same parameters, $t_{c^{\prime}}=8.9 \times 10^{6}$. Blue/red curves depict the numbers of atoms in (221) layers, $\widehat{N}_{\text {layer }}$, at $t=t_{a}$ (the left ordinate scale), and $t=t_{b}$ (the right ordinate scale), respectively. The olive/red ellipse mark the proto-drops/necks that merge/disappear with time. (B) The combined shape of the neck region, developing in the center of the nanowire, at two different times $t_{a}$ (the transparent glass-like atoms) and $t_{b}$ (the olive atoms). The neck region is shown from different observation points; blue and red arrows point to the same atoms situated on the facets of (001)- and (111)-type, respectively. (C) The [320]-type orientation; $L=450$ and $d=11\left(N_{0} \approx 170 \times 10^{3}, N_{t} \approx 162 \times 10^{3}, R_{\text {eff }} \approx 5.4\right)$. Subparts (a) and (b) show the shape of a nanowire fragment and the corresponding distribution, $\widehat{N}_{\text {layer }}(x)$, respectively, at the time $t=4 \times 10^{6}$ MC steps. The insets in subpart (b) demonstrate nanodrops resulting from the break-up of the fragment. Subpart (c) illustrates a snapshot of the nanowire configuration at $t=11 \times 10^{6} \mathrm{MC}$ steps.

\section{Discussion and Conclusions}

The disintegration processes of nanowires and liquid jets are similar only in that, in both cases, morphological evolution leads to a decrease in surface energy. The peculiarities in the breakup of the nanowire are based on the factors that determine the moving 'effective mass', $1 / \hat{k}^{2}$, and 'driving force', $-\partial \Delta E_{\text {surf }} / \partial \Delta_{c m}$ - see the Introduction. Both these parameters are dictated by the orientation of the nanowire axis relative to the planes that form the equilibrium Wulff construction. At the nonlinear stage, it is these planes that determine the morphology of the neck slopes and the distribution of the surface energy density on the emerged proto-drops.

The anisotropy of surface energy extends a possible set of evolutionary trajectories for a nanowire with an internal crystalline structure. Naturally, in the pre-melting regime ${ }^{[56]}$, when only the internal atoms are ordered in a crystalline structure, the break-up of the nanowire is more like the disintegration of a liquid jet. However, at lower temperatures, the nanowire displays effects that are not inherent to the liquid jet. The brightest examples are the emergence of step/snake/zigzag-like surface perturbations in nanowires with (211)-type orientation and long-living 2-3-body dumbbells, primarily in nanorods with (100) and (111)type orientations (See Supplementary Section S1). 


\section{WILEY-VCH}

In some cases, the distributions of evaporation flow density and the reverse diffusional flows of free atoms along the surface may substantially affect the parameters of nanowire break-up. Disbalance between these flows at local regions (necks and 'bulbs') can either decrease or increase the wavelength of emerging surface perturbations (see Supplementary Section S1). These effects strongly depend on the surface morphology and the nanowire temperature.

The variety of 'faceting' cylindrical nanowires at the initial stage of disintegration, which is unnatural for liquid jets, also significantly affects their further evolution. For instance, in the [111] nanowire orientation, a hexahedron is formed from the (110) planes with higher density of surface energy, whereas the nanowire with the [211] / [321] orientation is 'flattened' by two facets (111) with minimal density of surface energy. In the first case, the facets (111)/(100), with lower surface energy density, 'unseal' all lateral faces and form neighboring neck slopes. In the second, the appearance of necks is only possible on the edges of the flattened nanowire, which reduces the level of correlations between the diffusion flows at different narrowing regions compared with integrated axisymmetric necks formed in the [111] / [100] nanowire orientation. Thus, the pair of [211] / [321] -orientations are selected, as a priority, for consideration in our work, since it is in these cases that the distinctively expressed anisotropy of the nanowire break-up has been most predictable on the basis of simple qualitative estimations.

In the disintegration of the nanowire, the role played by end-effects is extremely important (which is doubtless a non-linear effect for reasons discussed in Supplementary Section S3). A long nanowire cannot break in many places at the same time. It is thus inevitable that long fragments form, the dynamics of which is affected by surface perturbations generated by their ends. In turn, the dynamics of the ends themselves, to a large extent (unlike liquid needles), depends on the morphology of the surface energy anisotropy, as far as fragments of facets of 


\section{WILEY-VCH}

different types form the scaly structure of lateral nanowire surfaces. This structure determines the rate of surface diffusion along the nanowire and the rate of detachment-reattachment processes providing additional diffusion of free atoms in the near-surface layer.

Thus, at a given temperature and for a given nanowire diameter, multiplicity arises in the values of parameter $\Lambda / r_{0}$ and the disintegration time. Additionally, ambiguities arise in the dispersion of the droplet size distribution and the appearance of surface perturbations in the form of bends/fractures, as observed in experiments, which can occur even in homogeneous nanowires without any defects. All aforementioned effects are defined by the anisotropy of the surface energy, which manifests itself differently depending on the nanowire orientation. On the other hand, the possibility of controlling this orientation provides a tool for the controlled spontaneous nanowire break-up/instability as one is able to estimate the orientation of 'main' crystal facets (i.e., the Wulff construction planes) relative to the nanowire axis and to analyze the structure of lateral surfaces. This allows predicting different break-up scenarios and determining associated parameters for technical applications.

The results obtained correlate well with the data acquired by real experiments and, most importantly, expound and interpret the physical mechanisms of various observed features in the break-up dynamics, which are associated with the anisotropy of surface physical properties in nanowires. 


\section{WILEY-VCH}

\section{Supporting Information}

Supporting Information is available from the Wiley Online Library or from the author.

\section{Acknowledgements}

We wish to thank Prof. S. Tretiak of the Center for Nonlinear Studies at Los Alamos National Laboratory and Prof. V. Tsifrinovich of New York University for having read through the manuscript and for the insight they provided. We are also thankful to Dr. D. S. Sultan for his useful comments on the manuscript. V.N.G. would like to thank his late colleague Prof. V. Privman of Clarkson University for the fruitful discussions they had in the course of analysis of the data and preparation of the manuscript.

\section{Conflict of Interest}

The authors declare no conflict of interest.

Received: ((will be filled in by the editorial staff))

Revised: ((will be filled in by the editorial staff)) Published online: ((will be filled in by the editorial staff))

\section{References}

[1] J.-H. Ahn, S.-J. Choi, J.-W. Han, T. J. Park, S. Y. Lee and Y.-K. Choi, Double-gate nanowire field effect transistor for a biosensor. Nano Lett. 10, 2934-2938 (2010).

[2] J. C. Claussen, A. D. Franklin, A. ul Haque, D. M. Porterfield and T. S. Fisher, Electrochemical Biosensor of Nanocube-Augmented Carbon Nanotube Networks. ACS Nano. 3, 37-44 (2009).

[3] Q. Wang, F. Min, J. Zhu, Preparation of gold nanowires and its application in glucose biosensing. Mater. Lett. 91, 9-11 (2013).

[4] Z. Xue, T. Dong, Z. Zhu, Y.Zhao, Y. Sun, Linwei Yu, Engineering in-plane silicon nanowire springs for highly stretchable electronics. J. Semicond. 39, N1. (2018).

[5] K. Zhang, Z. L.Wang, Y. Yang, Enhanced P3HT/ZnO Nanowire Array Solar Cells by Pyrophototronic Effect. ACS Nano. 10, 10331-10338 (2016).

[6] Y. Yin, Y. Sun, M. Yu, X. Liu, T. Jiang, B. Yang, D. Liu, S. Liu and W. Cao, ZnO Nanorod Array Grown on Ag Layer: A Highly Efficient Fluorescence Enhancement Platform. Sci. Rep. 5, 8152 (2015).

[7] H. S. Wasisto, S. Merzsch, A. Stranz, A. Waag, E. Uhde, T. Salthammer, E. Peiner, Silicon nanowire resonators for aerosol nanoparticle mass sensing. The 8th Annual IEEE International Conference on Nano/Micro Engineered and Molecular Systems, 506-509 (2013)

[8] C. M. Lieber, Building a Big Future from Small Things. MRS Bull. 28, 486-491, (2003).

[9] J. Naik, K. Das, P. Prewett, A. Raychaudhuri, Y. Chen, (2012). Liquid-like instabilities in gold nanowires fabricated by focused ion beam lithography. Applied Physics Letters. 101, (2012).

[10] H. Li, Hongwei, M. J. Biser, Jeffrey, T. J. Perkins, S. Dutta, P. R. Vinci, M. H. Chan, Thermal stability of $\mathrm{Cu}$ nanowires on a sapphire substrate. Journal of Applied Physics. 103, (2008).

[11] F. Granberg, S. Parviainen, F. Djurabekova, K. Nordlund, Investigation of the thermal stability of $\mathrm{Cu}$ nanowires using atomistic simulations. Journal of Applied Physics. 115, (2014). 


\section{WILEY-VCH}

[12] M. Rauber, F. Muench, M. E. Toimil-Molares, W. Ensinger, Thermal stability of electrodeposited platinum nanowires and morphological transformations at elevated temperatures, Nanotechnology. 23, N47, (2012).

[13] A. Volk, D. Knez, P. Thaler, A. W. Hauser, W. Grogger, F. Hofer, W. E. Ernst, Thermal instabilities and Rayleigh breakup of ultrathin silver nanowires grown in helium nanodroplets. Phys. Chem. Chem. Phys. 17, 24570-24575 (2015).

[14] N. E. Hjerrild, Darren C. J. Neo, A. Kasdi, H. E. Assender, J. H. Warner, Andrew A. R. Watt, Transfer Printed Silver Nanowire Transparent Conductors for PbS-ZnO Heterojunction Quantum Dot Solar Cells. Appl. Mater. Interfaces. 7, 6417-6421 (2015).

[15] S. P. A. Gill, Controlling the Rayleigh instability of nanowires. Appl. Phys. Lett. 102, 143108 (2013);

[16] S. A. Maier, P. G. Kik, H. A. Atwater, S. Meltzer, E. Harel, B. E. Koel, Ari A.G. Requicha, Local detection of electromagnetic energy transport below the diffraction limit in metal nanoparticle plasmon waveguides. Nature Materials. 2, 229-232 (2003).

[17] Z. Xue, M. Xu, Y. Zhao, J. Wang, X. Jiang, L. Yu, J. Wang, J. Xu, Y. Shi, K. Chen, P. Roca i Cabarrocas, Engineering island-chain silicon nanowires via a droplet mediated Plateau-Rayleigh transformation. Nat. Commun. 7, 12836 (2016).

[18] J. Naik, P. Prewett, K. Das, A. Raychaudhuri, Instabilities in Focused Ion Beam-patterned Au nanowires. Microelectronic Engineering. 88, 2840-2843 (2011).

[19] C.-H. Zhang, F. Kassubek, C. A. Stafford, Surface fluctuations and the stability of metal nanowires. Phys. Rev. B, 68, 165414-1-165414-8 (2003).

[20] J. Plateau, Experimental and Theoretical Statics of Liquids Subject to Molecular Forces Only, 1 (Gauthier-Villars, Paris, 1873).

[21] Lord Rayleigh, F.R.S., On the instability of jets. Proceedings London Mathematical Society, 10, 4-13 (1878).

[22] P. García-Mochales, R. Paredes, S. Peláez, and P. A. Serena, Statistical Molecular Dynamics Study of (111) and (100) Ni Nanocontacts: Evidences of Pentagonal Nanowires. Journal of Nanomaterials 2008, 361464 (2008).

[23] D. Min and H. Wong, Rayleigh's instability of Lennard-Jones liquid nanothreads simulated by molecular dynamics. Phys. Fluids 18, 024103 (2006).

[24] F. A. Nichols, W. W. Mullins, Surface- (interface-) and volume-diffusion contributions to morphological changes driven by capillarity. Trans. Metal. Soc. AIME 233, 1840-1848 (1965).

[25] S. Vigonski, V. Jansson, S. Vlassov, B. Polyakov, E. Baibuz, S. Oras, A. Aabloo, F. Djurabekova and V. Zadin, Au nanowire junction breakup through surface atom diffusion, Nanotechnology 29, Number 1 (2017).

[26] M. E. Toimil Molares, A. G. Balogh, T. W. Cornelius, R. Neumann, and C. Trautmann, Fragmentation of nanowires driven by Rayleigh instability. Appl. Phys. Lett. 85, 5337-5339 (2004).

[27] S. Karim, M. E. Toimil-Molares, A. G. Balogh, W. Ensinger, T. W. Cornelius, E. U. Khan, and R. Neumann, Morphological evolution of $\mathrm{Au}$ nanowires controlled by Rayleigh instability. Nanotechnology 17, 5954-5959 (2006).

[28] S. Karim, M. E. Toimil-Molares, W. Ensinger, A. G. Balogh, T. W. Cornelius, E. U. Khan, and R. Neumann, Influence of crystallinity on the Rayleigh instability of gold nanowires. J. Phys. D 40, 3767-3770 (2007).

[29] J.D. Powers, A.M. Glaeser, Orientation effects on the high-temperature morphological evolution of pore channels in sapphire. J. Am. Cer. Society 83, 2297-2304 (2004).

[30] V. Gorshkov, A. Zavalov, V. Privman, Shape Selection in Diffusive Growth of Colloids and Nanoparticles. Langmuir 25, 7940-7953 (2009). 


\section{WILEY-VCH}

[31] N. Gopan, S. P. Sathian, Rayleigh instability at small length scales. Phys. Rev. E 90, 033001 (2014).

[32] C. Zhao, J. E. Sprittles, and D. A. Lockerby, Revisiting the Rayleigh-Plateau instability for the nanoscale. J. Fluid Mech. 861, R3, (2019).

[33] J. Eggers, E. Villermaux, Physics of liquid jets. Rep. Prog. Phys. 71, 036601 (2008).

[34] T. Driessen, R. Jeurissen, H. Wijshoff, F. Toschi, and D. Lohse, Stability of viscous long liquid filaments. Phys. Fluids 25, 062109 (2013).

[35] J. Eggers, Dynamics of liquid nanojets. Phys. Rev. Lett. 89, 084502, (2002).

[36] A. J. Bernoff, A. L. Bertozzi, and T. P. Witelski Axisymmetric Surface Diffusion: Dynamics and Stability of Self-Similar Pinchoff. Journal of Statistical Physics 93, 725-776 (1998).

[37] Weeks J.D. (1980) The Roughening Transition. In: Riste T. (eds) Ordering in Strongly Fluctuating Condensed Matter Systems. NATO Advanced Study Institutes Series (Series B: Physics), vol 50. Springer, Boston, MA. DOI https://doi.org/10.1007/978-1-4684-3626-6_21

[38] M. J. Rost, S. B. van Albada, J. W. M. Frenken, Thermally activated domain boundary formation on a missing row reconstructed surface: Au(1 10$)$. Surf.Sci. 547, 71-84 (2003).

[39] D.A. King, D.P. Woodruff, Phase Transitions and Adsorbate Restructuring at Metal Surface. Elsevier Science 7, (1994).

[40] V. Gorshkov, V. Privman, Models of Synthesis of Uniform Colloids and Nanocrystals, Physica E 43, $1-12(2010)$

[41] V. Gorshkov, O. Zavalov, P. B. Atanassov, V. Privman, Morphology of Nanoclusters and Nanopillars Formed in Nonequilibrium Surface Growth for Catalysis Applications. Langmuir 27, 8554-8561 (2010).

[42] V. Privman, V. Gorshkov, and O. Zavalov, Formation of Nanoclusters and Nanopillars in Nonequilibrium Surface Growth for Catalysis Applications: Growth by Diffusional Transport of Matter in Solution Synthesis. Heat Mass Transfer 50, 383-392 (2014).

[43] V. Gorshkov, V. Kuzmenko, V. Privman, Nonequilibrium Kinetic Modeling of Sintering of a Layer of Dispersed Nanocrystals. CrystEngComm 16, 10395-10409 (2014).

[44] V. Gorshkov, V. Kuzmenko, and V. Privman, Mechanisms of Interparticle Bridging in Sintering of Dispersed Nanoparticles. J. Coupled Syst. Multiscale Dynamics 2, 91-99 (2014).

[45] V. Privman, V. Gorshkov, Y. E. Yaish, Kinetics Modeling of Nanoparticle Growth on and Evaporation off Nanotubes. J. Appl. Phys. 121, 014301 (2017).

[46] V. Gorshkov, V. Privman, Kinetic Monte Carlo model of breakup of nanowires into chains of nanoparticles. Journal of Applied Physics 122, 204301 (2017).

[47] D. Foster, R. Ferrando, R. Palmer, Experimental determination of the energy difference between competing isomers of deposited, size-selected gold nanoclusters. Nature Communications 9, 1323 (2018).

[48] V. N. Gorshkov, M. G. Chaban, Nonlinear electrohydrodynamic phenomena and droplet generation in charged jets of conducting liquid. Technical Physics 44, 1259-1266 (1999).

[49] I. Sevonkaev, V. Privman, and D. Goia, Synthesis of dispersed metal particles for applications in photovoltaic, catalysis and electronics. J. Solid State Electrochem 17, 279-297 (2012).

[50] K. Balantrapu, M. McMurran, and D. V. Goia, Inkjet printable silver dispersions: formation and electrical conductivity. J. Mater. Res. 25, 821-827 (2010).

[51] G. Wulff, Zur frage der geschwindigkeit des wachstums und der auflosung der kristallflachen. $Z$. Kristall. Mineral. 34, 449 (1901).

[52] C. Herring, In Structure and Properties of Solid Surfaces, R. Gomer, C. S. Smith, Eds. (University of Chicago Press: Chicago, 1953) Chapter 1. 


\section{WILEY-VCH}

[53] J.K.Mackenzie, A.J.W.Moore, J.F.Nicholas, Bonds broken at atomically flat crystal surfaces-I: Facecentred and body-centred cubic crystals. J. Phys. Chem. Solids 23, 185-196 (1962).

[54] J. A. Venables, Introduction to Surface and Thin Film Processes. Cambridge University Press: Cambridge, U.K., 2000.

[55] Gorgios D. Barmparis, Zbigniew Lodziana, Nuria Lopez, Ioannis N. Remediakis, Nanoparticle shapes by using Wulff constructions and first-principles calculations. Beilstein J. Nanotechnol. 6, 361-368 (2015).

[56] A. Dutta, S. Chatterjee, A. K. Raychaudhuri, A. Moitra, and T. Saha-Dasgupta, In-silico investigation of Rayleigh instability in ultra-thin copper nanowire in premelting regime. J. Appl. Phys. 115, 2443031 (2014).

[57] K. C. Lai, J. W. Evans, Reshaping and sintering of 3D fcc metal nanoclusters: Stochastic atomistic modeling with realistic surface diffusion kinetics. Phys. Rev. Materials 3, 026001 (2019). 


\section{WILEY-VCH}

The effects of the nanowire orientation relative to the axes of symmetry of its internal crystal structure on its disintegration into nanoclusters is studied. A qualitative interpretation is given for the variety of the quantitative characteristics of the breakup observed in experiments. The contribution, to the classical Plateau-Rayleigh instability, of factors that are characteristic only for crystalline 'jets' (anisotropy of the surface energy, thermal roughening, long-lived unduloid-like configurations) is studied.

Keyword ((Plateau-Rayleigh instability, FCC lattice structure, anisotropy, break-up, nanowire, nanodrop))

Vyacheslav N. Gorshkov, Pooya Sareh*, Vladimir V. Tereshchuk, Arash Soleiman-Fallah

Title ((Dynamics of anisotropic break-up in nanowires of FCC lattice structure))
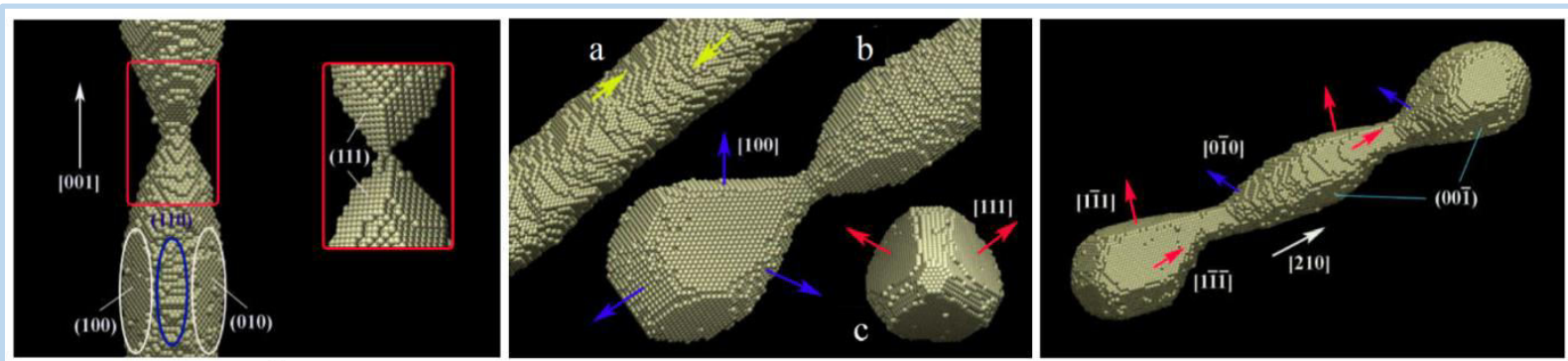

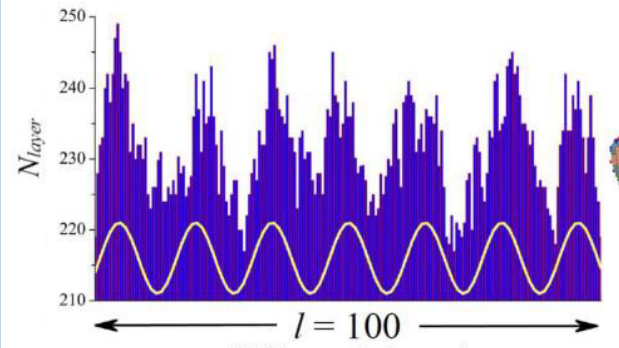

(200 atomic layers)
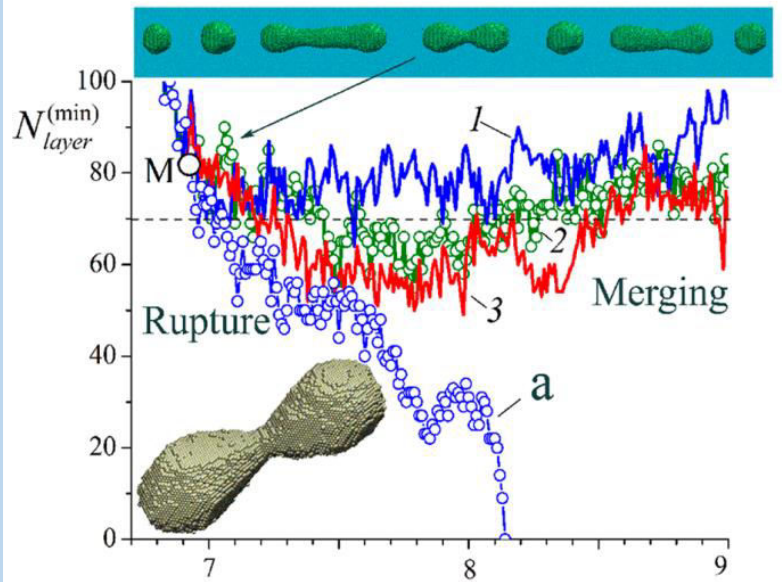

$t / 10^{6} \mathrm{MC}$ time steps
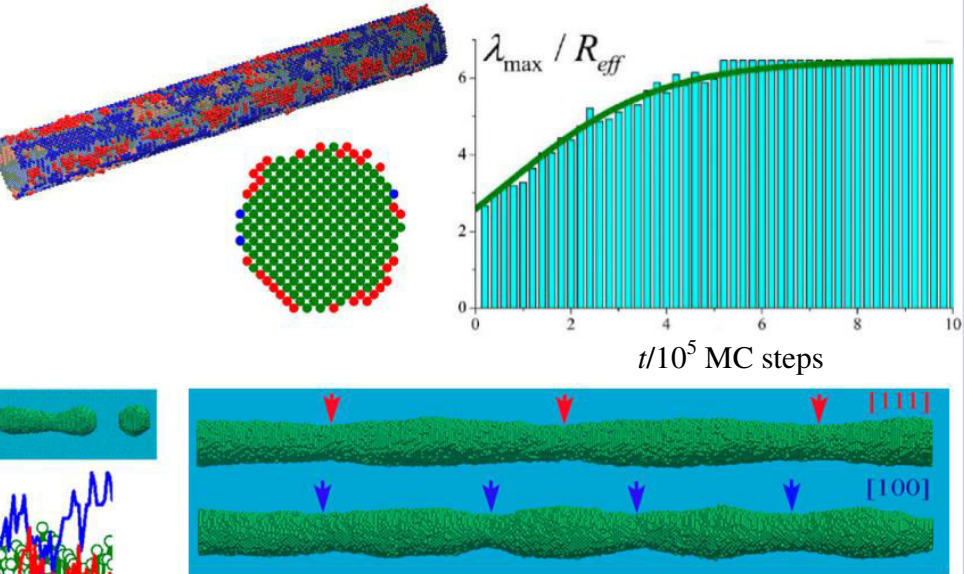

a

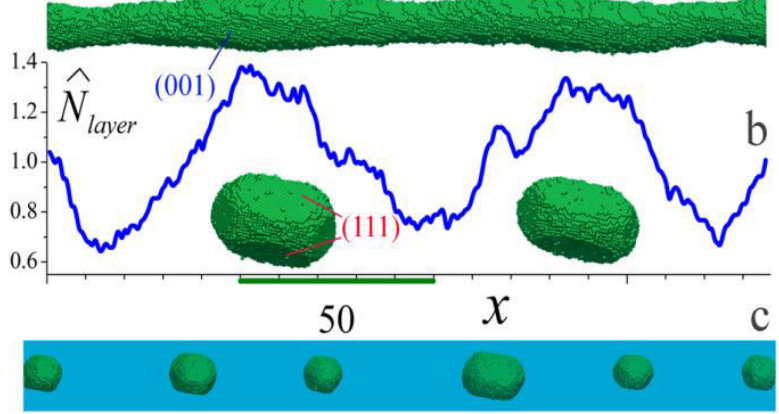

ToC Figure 


\title{
WILEY-VCH
}

Copyright WILEY-VCH Verlag GmbH \& Co. KGaA, 69469 Weinheim, Germany, 2018.

\section{Supporting Information}

\author{
Dynamics of anisotropic break-up in nanowires of FCC lattice structure
}

Vyacheslav N. Gorshkov, Pooya Sareh*, Vladimir V. Tereshchuk, Arash Soleiman-Fallah

\section{Supporting Sections}

\section{Section S1. Dynamics of nanowires with rotational symmetry}

In the course of the break-up of nanowires with [100]- and [111]-type orientations (Movies S1-S4), their axes, with a good approximation, are the axes of rotational symmetry of the fourth- and third-order, respectively. In such cases, the results obtained by existing analytical models of instability ${ }^{[20,24]}$ and some physical experiments correlate strongly (including for the value of the break-up parameter $\Lambda / R_{e f f}$ ). Recall that $R_{e f f}$ is the nanowire radius when a steady equilibrium state between the nanorod and the vapor of free atoms, which fill the surrounding container bounded by the reflecting walls, is established. We note that the equilibrium is achieved at a time which is much less than the time needed for the break-up to occur. If the initial number of atoms in the nanorod is $N_{0}$, and their number in the equilibrium state is $N_{t}$, then:

$$
R_{e f f} \approx r_{0} \sqrt{N_{t} / N_{0}}
$$

where $r_{0}$ is the initial radius of the nanowire.

In this study, we conducted a series of numerical simulations on nanowires with both 'free' and 'frozen' ends. In the case of 'frozen' ends, during the formation of the initial state, 'extensions', with the width of five atomic layers, are attached to the ends of the wire. The atoms of these 'extensions' are treated as motionless hence the term 'frozen'. Generally, small fragments are formed in the end regions of the nanowire, and while estimating the parameter $\Lambda / R_{e f f}$ by the number of created droplets (or spaces between them), we consider the first and the last fragments as a single drop, as a realization of the periodic boundary conditions.

It is to be noted that the number of droplets formed in each numerical simulation is random. For [100]-type orientation (see Fig. S1, depicting that at the initial stage the nanowire is mostly bounded by four (100)- and four (110)- planes). The average number of drops (averaged over the results of ten random simulations of the break-up process) is between 8 and 8.3. In the case of the nanowire with length $L=450$, and $R_{e f f} \approx 6$, the break-up parameter varies in the range of $\Lambda / R_{\text {eff }} \in[9,9.3]$. Whereas, Fourier analysis of the distribution $N_{\text {layer }}(x)$ at the initial break-up stage (see configuration (b) in Fig. S1) gives that maximal amplitude has a mode with $\lambda_{\max } \approx 7.4 R_{\text {eff }}$. 


\section{WILEY-VCH}

A

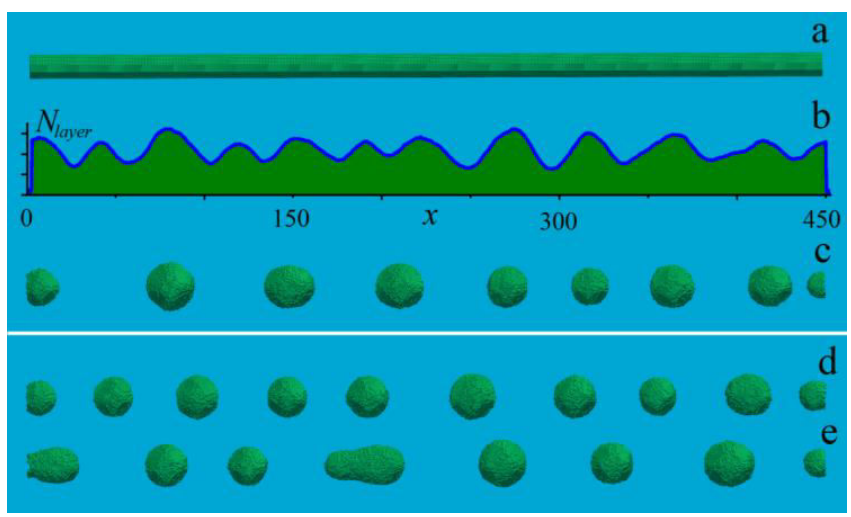

B

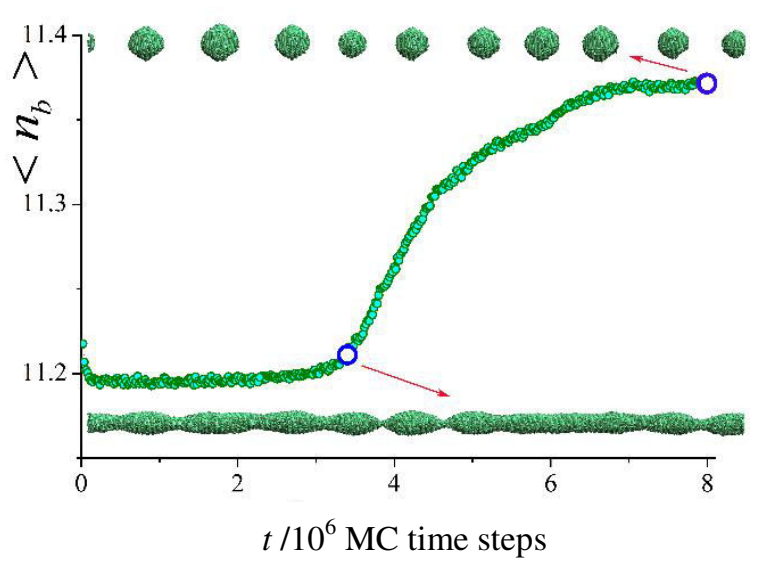

Fig. S1. Break-up of a nanowire with [100]-orientation and frozen ends in the warm regime: $\alpha=0.9, p=0.725, L=450$, and $d \approx 12.5$. (A) Configuration (a) shows the initial shape of the nanowire. Configuration (b) yields the number of particles in the (100)-type atomic layers at $t=2.3 \times$ $10^{6}$ (an average number of atoms in a layer $\sim 220$ ). Configuration (c) illustrates the state of the system at the last break-up stage, $t=7.2 \times 10^{6}$. Configurations (d) $\&$ (e) are the results of two other random MC simulations at $t=6.9 \times 10^{6}$ and $t=7.2 \times 10^{6}$, respectively. The initial number of particles in the nanowire equals approximately $\sim 215$ thousands while drops contain about $\sim 199$ thousands of atoms. (B) Typical dynamics of the average number of bonds per atom, $\left\langle n_{b}(t)\right\rangle$. The parameters of the nanowire are presented above.

The decrease in surface energy during the disintegration process can be partially described by an increase in the average number of bonds per atom, $\left\langle n_{b}(t)\right\rangle$ (See. Fig. S1B). One can see that it is at the stage of inititation of nanowire break-up that the most significant decrease in $\mathrm{E}_{\text {surf }}$ (i.e. growth of $\left.\left\langle n_{b}\left(t>3 \times 10^{6}\right)\right\rangle\right)$ takes place. A small decrease in $\left\langle n_{b}\left(t \lesssim 4 \times 10^{5}\right)\right\rangle$ from 11.216 to 11.193 at the initial stage, which is a result of the onset of thermal roughness, is followed by a long plateau-like dependence $\left\langle n_{b}(t)\right\rangle$. Thus, this parameter does not fully reflect the tiny details of the dynamics of the total surface energy, when long-wave perturbations of the surface prevail and the surface energy density is sufficiently inhomogneous along the nanowire.

This pattern of $E_{\text {surf }}(t)$ is observed for all crystallographic orientations of the nanowire discussed it this study. It should be noted that data presented on Fig. S1B demonstrate a rare case, when each bead in the string of beads before the onset of the break-up $\left(t \approx 3.3 \times 10^{6}\right)$, transformed into a single drop without mergence with neighboring beads as it often happens.

For the 'cold' regime (see Fig. S2) the nanowire radius was chosen so that, in the equilibrium state, the number of atoms in the nanorod was approximately the same as for the nanowire depicted in Fig. S1. It is important to point out, that the decrease in temperature led to a noticeable increase in the break-up time. Additionally, the formation of long-living 3-body dumbbells (as depicted by configuration (b) in Fig. S2) is typical in the "cold" regime and was already observed in real experiments ${ }^{[27,28]}$. The evolution of such fragments usually ends with break-up when one of the extreme particles absorbs the middle part. The break-up parameter averaged over 10 simulations was approximately $\frac{\Lambda}{R_{e f f}} \approx 9.3$. 


\section{WILEY-VCH}

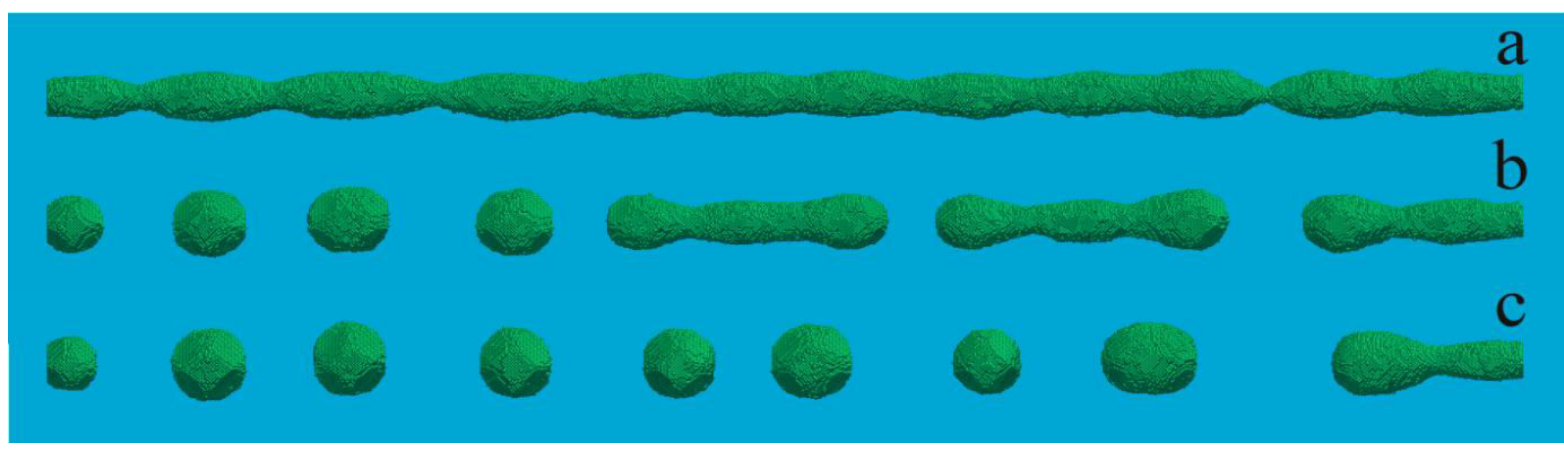

Fig. S2. Disintegration of a nanowire with its axis along the [100]-orientation of FCC in the cold regime: $\alpha=1.2, p=0.652, L=450, d \approx 12$. The number of atoms at the start of the process is $\sim 199$ thousands. Drops contain approximately $\sim 197.5$ thousand atoms. The snapshots of configurations (a), (b), and (c) are taken at $t=14,20,32\left(\times 10^{6}\right)$ MC time steps, respectively.

Different break-up scenarios of the nanowire with [111]-type orientation are shown in Fig. S3. We would like to note the durability of the 2-body rightmost dumbbell presented in Fig. S3A, subparts (c), (d), (e), and (f), while 3-body leftmost dumbbell broke up into two droplets despite its initially wider neck (see subpart (c) in Fig. S3A). Here, the break-up parameter $\Lambda / R_{\text {eff }}$ varies between 8.7 and 9.3. Thus, for the two orientations of the nanowire discussed, an average distance between nanoclusters after the break-up corresponds to the values most frequently mentioned in works of our predecessors ${ }^{[26-28]}$. However, we should again point out, that the obtained value of the parameter $\Lambda / R_{e f f}$ is a result of nonlinear effects and that shorter wavelength perturbations of the nanowire surface dominate at the linear stage.

Data presented in Fig. S3B give information regarding the role of surface diffusion of the bonded atoms and bulk diffusion of the evaporated ones. The diffusion of atoms in the nearsurface layer plays a significant role in the disintegration of the [111]-nanowire. If, in the numerical model, we block the sublimation of atoms from the surface of the nanowire (i.e. 'turn off' the bulk diffusion), then the break-up time will increase by more than five times (compare the data presented in Fig. S3A and Fig. S3B). Whereas, the same numerical experiment conducted with nanowires with [100]-type orientation gives an increase in the break-up time by only $20-30 \%$.

Data presented in Fig. S3C clearly demonstrate one more feature of the break-up of a nanowire with the [111]-orientation. When atoms from the surrounding vapor are deposited on the nanowire surface, the wavelength of the surface perturbations is noticeably shorter (see Fig. $\mathrm{S} 3 \mathrm{~A}(\mathrm{~b}), \lambda_{\max } / R_{\text {eff }} \approx 6.9$ ), than in the case with blocked evaporation (see Fig. S3C, $\lambda_{\text {max }} / R_{\text {eff }} \approx 10$ ). This means that in the first case the well-known effect arises when the diffusion flows from surrounding space to a surface can result in the formation of shortwave perturbations on it if the surface diffusion is low. However, this effect does not appear in the [100]-orientation where the surface diffusion dominates the formation of the sausage-like surface perturbations (in both cases (Fig. S1B and Fig. S3C) $\lambda_{\max } / R_{\text {eff }} \approx 6.9$ ). Thus, the sensitivity of the nanowire dynamics to the surrounding vapor density suggests that the temperature dependency of the breakup parameter, $\Lambda / R_{\text {eff }}$, is mainly inherent to the [111]nanowire orientation, as observed for $\mathrm{Cu}^{[26]}$. The results discussed once again confirm the important role of nanowire orientation, which determines the anisotropy of (i) the surface energy density, (ii) the coefficient of surface diffusion, and (iii) the local rates of sublimation. 


\section{WILEY-VCH}
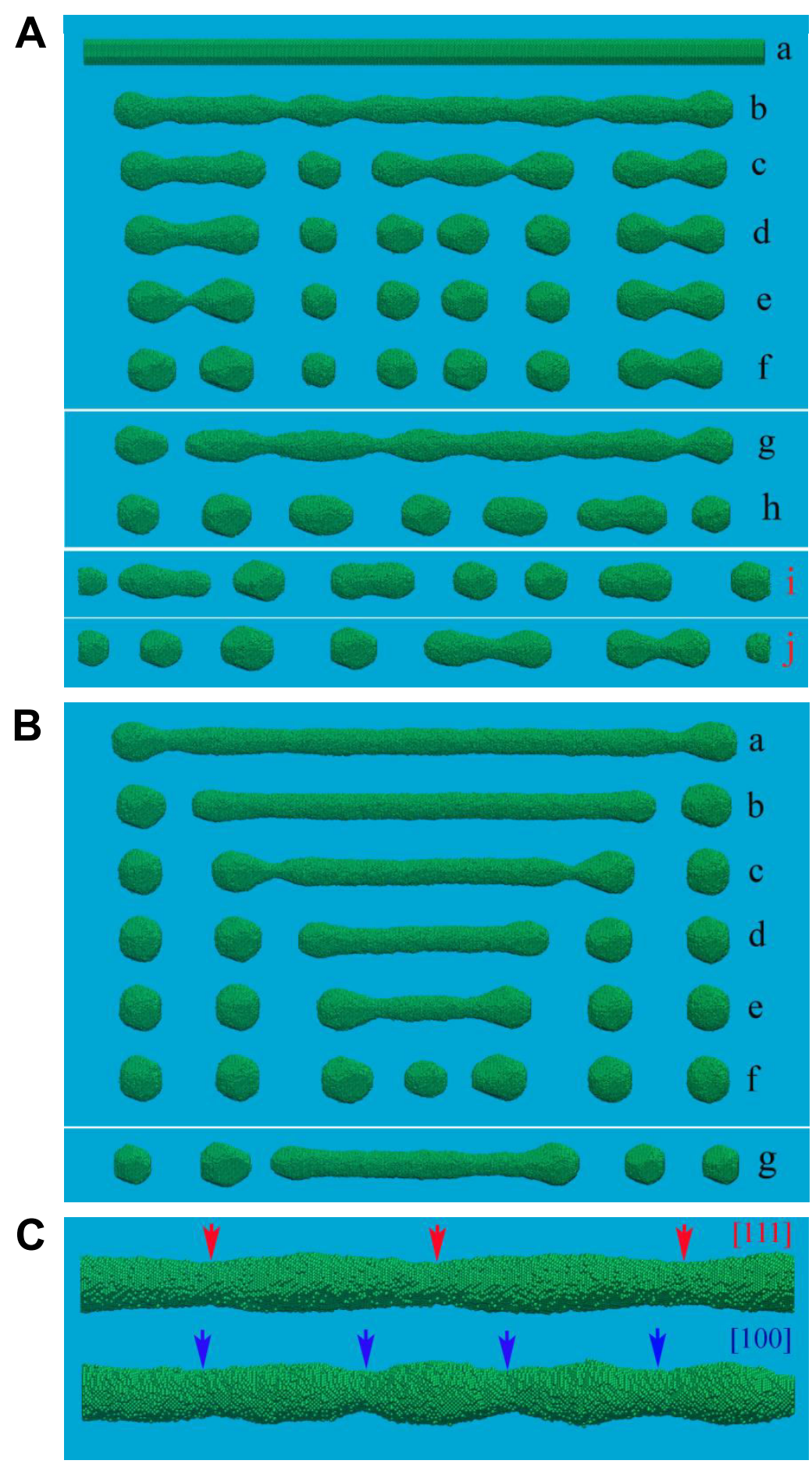

Fig. S3. Break-up processes of nanowires with [111]- orientation with both free and frozen ends. (A) Warm regime: $\alpha=0.9, p=0.725, L=360, d \approx 12.5$. Subpart (a) depicts the initial shape of the nanowire; subparts (b) to (f) show the break-up process at $t=\{2.5,3.5,4.5,5.5,6.6\} \times 10^{6}$, respectively. At the start of the process, the nanowire consists of $\sim 191$ thousand atoms while drops have $\sim 170$ thousand atoms. Subparts (g) and (h) depict the result of another random simulation of the break-up; the snapshots are taken at $t=3.5 \times 10^{6}$ and $4 \times 10^{6} \mathrm{MC}$ time steps, respectively. Subparts (i) and (j) show the final configurations of the nanorod with 'frozen' ends in two random numerical simulations. The snapshots are taken at $t=4.56 \times 10^{6}$ for (i) and $t=4.8 \times 10^{6}$ for (j). The first break-ups occurred at $t=2.1 \times 10^{6}$ (i) and $t=3.3 \times 10^{6}$ (j). (B) Warm regime with blocked sublimation, $L=360, d \approx 12$. Only end-mechanism takes place. The snapshots of configurations (a) to (f) were taken at $t=\{4,6,10,12,16,18\} \times 10^{6}$, respectively. The system contains $\sim 170$ 


\section{WILEY-VCH}

thousands of atoms, which is the same number as in the nanorod depicted in Fig. S3A after the equilibrium of the nanowire with the surrounding vapor is established. Subpart (g) depicts the cold regime: $\alpha=1.2, p=0.652, t=28.5 \times 10^{6}$; the evaporation process is included. (C) Snapshots of nanowire fragments with [111] and [100] orientations and length $L_{f r}=175$ during the disintegration process with blocked evaporation at $t=15 \times 10^{6}$ and $t=3 \times 10^{6} \mathrm{MC}$ time steps, respectively.

The physical mechanism of the occurrence of such distinction is quite easy and takes place due to the nanowire surface structure (see. subpart (a) in Fig. 1B in the main text). An atom, moving on fragments of filled (111)-type surfaces, has the lowest possible binding energy, therefore the probability of detachment from the surface is high. Furthermore, a transition from one fragment to another is associated with a high probability for an atom to be trapped by corner-surface-vacancies with high binding energy. Therefore, 'turning off' the bulk diffusion noticeably suppresses the development of instabilities on the nanowire surface. Instead, end-effects dominate during the disintegration process (see subparts (a) to (f) of Fig. S3B, and Movies S1 and S2). The enhancement of the role played by end-effects with decreasing temperature is also predictable (as depicted by subpart (g) of Fig. S3B): suppressing the evaporation of atoms from the surface (bulk diffusion) reduces the increment of amplification of thermal fluctuations. At the same time, modulations of the radius in the case of [100]-type orientation are clearly visible (as shown in configuration (a) of Fig. S2) before the break-up.

The comparison of the results for [100] and [111] orientations demonstrates that surface diffusion plays a dominant role in the first case whereas bulk diffusion, in the second. However, the differences in the values of the parameter $\Lambda / R_{e f f}$ are insignificant $\left(\Lambda / R_{e f f} \approx\right.$ $9 \pm 0.3)$.

\section{Section S2. Internal atom diffusion}

In this section, we will discuss the mechanism of internal atom diffusion since it may not be explained, likewise, within the framework of existing models ${ }^{[24]}$. For this purpose, we conducted a numerical simulation with a cylindrical (111) nanorod ( $L=250$ and $d=16$ ). Atoms originally in the central (111) layer (at $t=0$ ) are marked blue. After $t=2 \times 10^{5} \mathrm{MC}$ time steps (we note that the average break-up time of the nanowire under consideration is at least 50 times greater than the indicated time interval) the distribution of marked atoms in adjacent (111)-type atomic layers was studied (see Fig. S4 - the number of the central layer is assumed to be zero). It is worth mentioning that at this stage more than $86 \%$ of marked atoms have left the central layer (see Fig. S4A). The same simulations were carried out for nanowires with a [100]-type orientation. The mechanisms of the observed bulk diffusion are physically transparent. After the step-like structure on the lateral surface of the nanowire is formed (see, for instance, subpart (a) in Fig. 1B) the corner surface vacancy can be occupied by an internal atom, so a vacancy will appear within the nanowire (see Fig. S4B and Fig. $\mathrm{S} 4 \mathrm{C}$ ). The drift of such vacancies in the volume of the wire leads to a noticeable mixing of internal atoms and contributes to the reduction of the parameter $\left\langle n_{b}(t)\right\rangle$ (see Fig. S1B) at the initial stage. For the nanowire presented in Fig. S1B and Fig. S4C, the difference $\Delta n_{b}=$ $\left\langle n_{b}(0)\right\rangle-\left\langle n_{b}\left(t=4 \times 10^{5}\right)\right\rangle$, is provided by these internal vacancies up to $\Delta n_{b} / 4$ (the other part, i.e. $3 \Delta n_{b} / 4$, is a result of the thermal roughening of the surface).

An analogous effect is also observed in nanoparticle sintering [43,44] where there is no contact between nanoclusters at the point of initiation of the process. Atoms of one nanocluster left their initial positions and deposited on the surface of a neighboring cluster as 


\section{WILEY-VCH}

a result of detachment-reattachment processes, creating surface roughness and 'active' vacancies in the near-surface layers of the nanocluster. The drift of such vacancies led to the migration and mixing of 'foreign' atoms with atoms of the neighboring cluster.

The role played by volume diffusion in the break-up process of nanowires, as described earlier, requires further investigation. However, we can expect its noticeable contribution to the dynamics of the process just before the break-up since in the neck region, the ratio of surface area to volume increases. As a result, the bulk concentration of active vacancies in narrow regions of a nanowire is higher than in wide ones. The predominant drift of vacancies from 'narrow' to 'wide' regions corresponds to reverse flow of atoms to the locations of potential rupture in the nanowire and may cause changes in the statistical characteristics of the process. 


\section{WILEY-VCH}

A

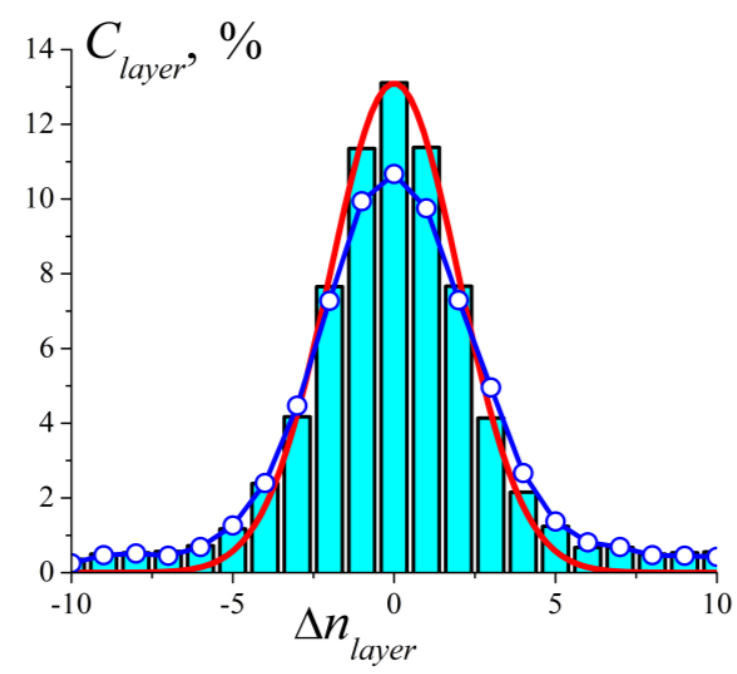

B

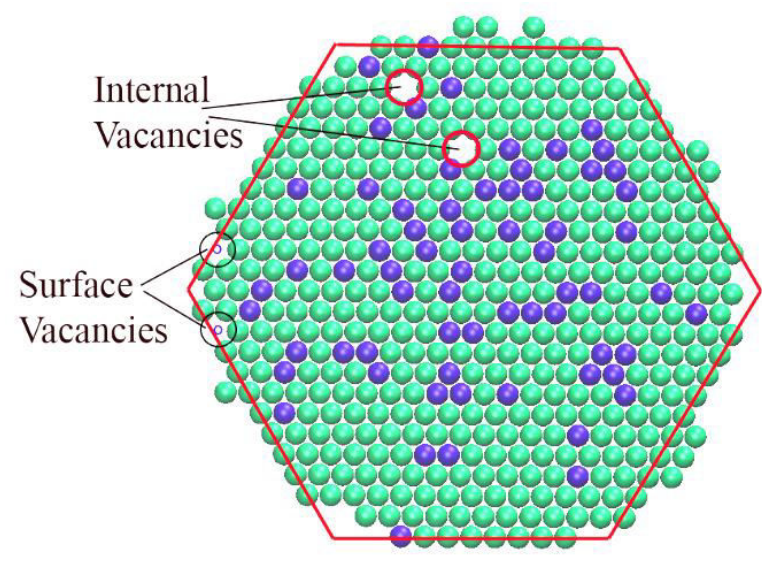

\section{C}

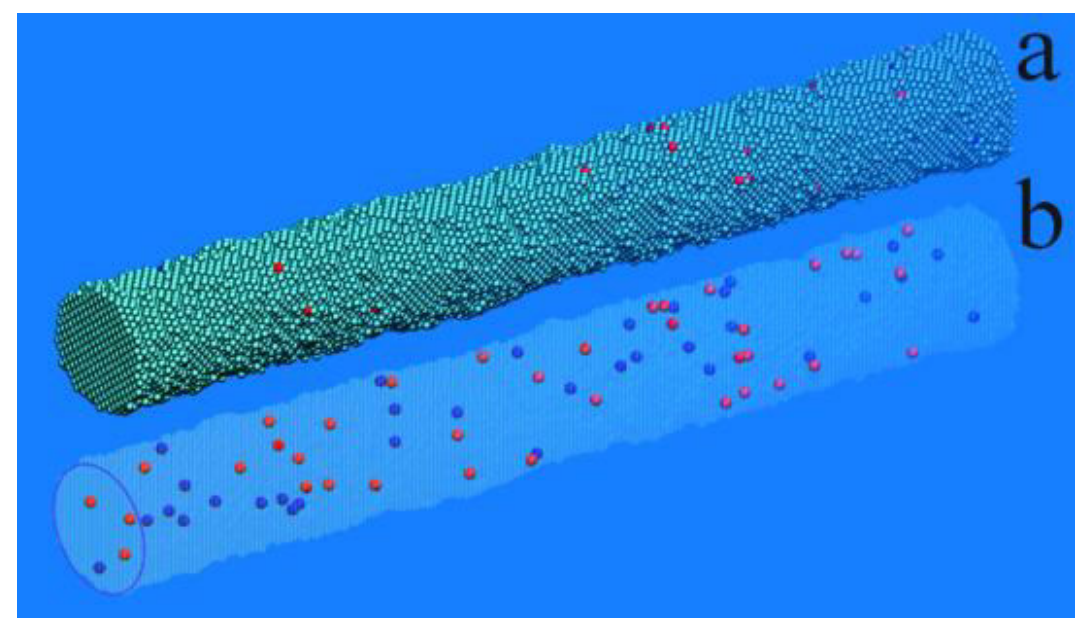

Fig. S4. Illustration of the internal fluxes of atoms in the nanowire in the warm regime. $(\alpha=$ $0.9, p=0.725$, with model parameters $L=250$, and $d=16$ (6.5 $\mathrm{nm}$ in the case of Au), $t=2 \times$ $10^{5} \mathrm{MC}$ steps). (A) Cyan bars show the distribution of marked atoms in the layers of a nanowire with (111)- type orientation. An average number of atoms in cross-section $\left\langle N_{\text {layer }}\right\rangle=470, C_{\text {layer }}=$ $N_{\text {marked }} /\left\langle N_{\text {layer }}\right\rangle$. First eleven layers closest to the center, $\Delta n_{\text {layer }} \in[-5,5]$, contain $66 \%$ of all marked atoms, $72 \%$ are situated within $[-10,10]$ range, and the region with $\left|\Delta n_{\text {layer }}\right|>50$ contains $11 \%$ of atoms. If detachment is blocked, then in the regions $\left(-10 \leq \Delta n_{\text {layer }} \leq 10\right)$ and $\left|\Delta n_{\text {layer }}\right|>$ $50,77 \%$ and only $2 \%$, of marked atoms are contained, respectively. Red fitted curve is an approximation function. $C_{\text {layer }}=13.1 \times \exp \left(-\Delta n_{\text {layer }}^{2} / 8\right)$. Blue circles and a curve - represent the result for nanowires with (100)-type orientation. Each atomic layer of the nanowire with (100)-type orientation has approximately 420 atoms. (B) The atomic distribution in the central layer ([111]orientation) after $t=2 \times 10^{5} \mathrm{MC}$ time steps. Violet and green spheres show marked and extrinsic atoms, respectively. (C) A fragment of a long nanowire with [100]-orientation at time $t=4 \times 10^{5}$ (the 'warm' regime, length of the fragment $L_{f r}=100$, and $d \approx 12$ ). Metallic pastel spheres in (a) and glass spheres in (b) represent the bonded atoms. Red spheres are the surface vacancies which have the nine nearest lattice points occupied, $m_{v a c}=9$; for 'blue' vacancies $10 \leq m_{v a c} \leq 12$. 


\section{WILEY-VCH}

\section{Section S3. End-effects in the nanowire break-up}

As already mentioned, it would be incorrect to consider the break-up of a finite nanowire (as well as of a liquid thread) as a process of developing instability since there is no equilibrium state associated with it. In an infinite nanowire, the break-up starts with an increase in thermal fluctuations with time, however; we point out that the nanowire end itself excites much larger fluctuations propagating to its interior region (see Fig. S5B).

The wavelength of such fluctuations, as for finite liquid streams ${ }^{[20]}$, is of the order of the nanorod diameter, if the nanowire is aligned along the [100] or [111]-axis. The physical mechanisms of the process are also similar, though in the case discussed we examine crystalline structure without intense internal flows. Let us perform a qualitative analysis of the images shown in Fig. S5 without considering the anisotropy of surface energy. Rounding of nanowire end is associated with the surface outflow of atoms, $\Gamma^{(-)}$, from the regions with low binding energy, i.e. edges, fractures, and other areas of large positive curvature, to the base of the nanowire (see subpart (a) of Fig. S5A). The newly formed moving 'bulb' creates, in front of itself, an area of increased binding energy (i.e. a zone of negative mean surface curvature), initiating counter flows of atoms $\Gamma^{(+)}$, which leads to the neck formation in front of the bulb (as depicted in subparts (a) and (b) of Fig. S5A). The back of the forming constriction has an additional positive curvature of the surface compared to the unperturbed part of the nanorod, which is the cause for the excitation of new flows, $\Gamma^{(-)}$, directed to the base of a nanowire (see subpart (c) of Fig. S5A). The dynamics of change in the number of particles in the (111)type atomic layers (Fig. S5B) visually represents the result of the processes taking place.

The surface perturbations, excited by nanowire ends, can interfere with each other (if a nanowire is relatively short) and with increasing thermal fluctuations of the central part of longer nanorods. We note that the role of end-effects in the nanowire break-up increases with the temperature reduction (i.e. reduction in thermal fluctuations) and that intense surface diffusion prevents the formation of bulbs at the nanowire ends and suppresses end-effects. 


\section{WILEY-VCH}

A
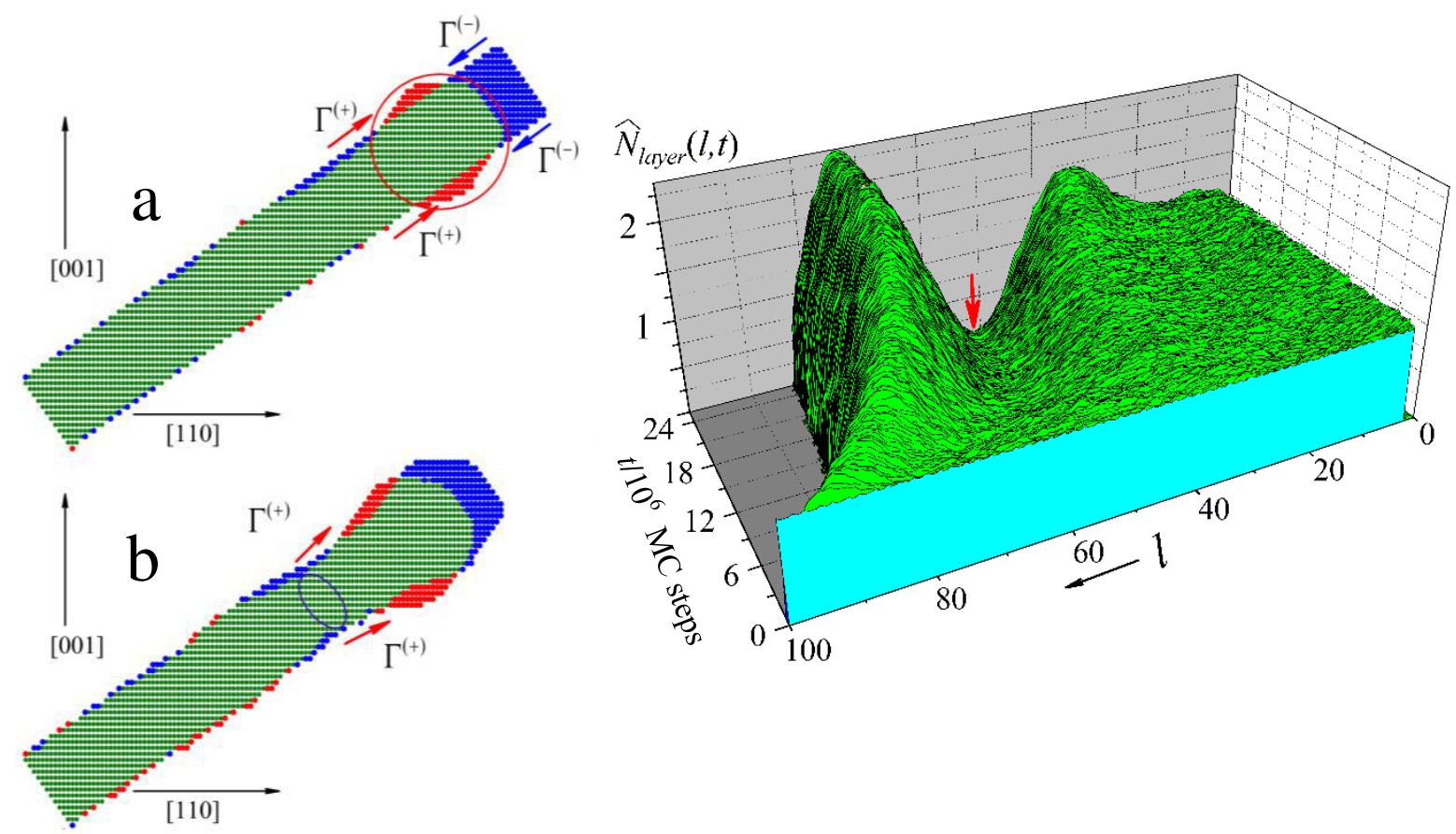

B

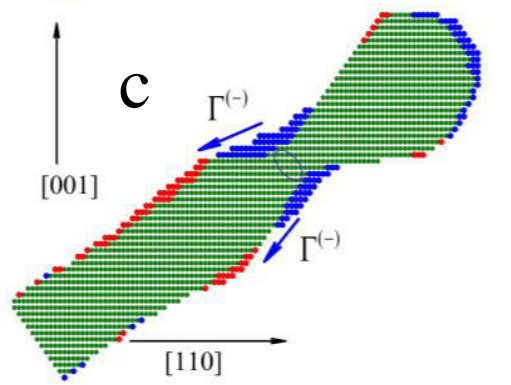

Fig. S5. Evolution of a nanowire end with its axis aligned along the [111]-type orientation of FCC. The result was obtained for reduced temperature regime: $\alpha=1.2, p=0.65$, cross section size $d=14$, and initial length $L=100$. The first five atomic layers from the left were 'frozen', so the evolution of nanorods depends only on the processes at the upper end. (A) Changes in the atomic distribution on the (-110)-type plane, which intersects the nanowire axis. Each snapshot was obtained by the combination of atomic distributions for two consecutive instants of time $t_{1}$ (olive + blue circles) and $t_{2}$, (olive + red circles), $t_{2}>t_{1}$; in other words, olive circles represent only unchangeable system of bounded atoms at the two subsequent moments. Subpart (a): $t_{1}=0, t_{2}=4 \times 10^{6}$; subpart (b): $t_{1}=4 \times 10^{6}, t_{2}=11 \times 10^{6}$, and subpart (c) $t_{1}=16 \times 10^{6}, t_{2}=24 \times 10^{6}$. The cut-off of a nanoparticle takes place at $t=25.18 \times 10^{6} \mathrm{MC}$ steps. The length of a residue $\approx 52$. (B) Dynamics of the number of atoms in (111)-atomic layers of this nanowire, $\widehat{N}_{\text {layer }}(l, t)=N_{\text {layer }}(l, t) / N_{\text {layer }}(t=$ $0), N_{\text {layer }}(t=0) \approx 360$. 


\section{WILEY-VCH}

\section{Section S4. Analysis of long-lived 2-3-body dumbbells}

The formation of long-lived 2-3-body dumbbells was observed in our numerical simulations of the nanowire break-up (see Figs. S6A and S6B) as well as in real experiments ${ }^{[12,27]}$, a phenomenon inexplicable on the basis of any hydrodynamic models. The results of calculation of the dynamics of a relatively short nanorod $\left(L / r_{0}=16\right)$ are presented below. We note that, in this case, applying the theory about preferable development of disturbances with wavelength $\lambda \approx 9 r_{0}$ is unfounded. In such situations, two-way end-effects take place (see S3) and the result of interference of two perturbations originated at each end is totally unpredictable. Among a great number of evolutionary 'trajectories' of the multi-particle system, we present only two simplest ones. The first one - nanowire breaks up into two drops for a relatively short time interval (Movie S5). The second one - the nanowire is 'trapped' in one of the local energy minima and stays in this state of quasi-stable equilibrium for a long time until thermal fluctuations take it out of such a state. Subsequently, an unpredictable outcome will follow again. Data presented in Fig. S6 (the results of two random simulations of the dynamics of the nanowire with the same values of parameters for both cases) demonstrate the details of such 'trajectories'. Fig. S6C demonstrates the formation of an unduloid-like dumbbell directly during the disintegration of a long nanowire. Different random realizations of its evolution at $t>6.9 \times 10^{6}$ correspond either to fast break-up or slow long-term merger.

Three-body dumbbells are less common, a phenomenon which is consistent with the principles of probability theory. Fig. S7 demonstrates the existence of a rare case (of low probability) of the formation of 3-body nano-objects in quasi-equilibrium. We indicate that the simulation was conducted at the 'cold' regime in order to reduce the influence of thermal fluctuations. This regime is dictated by the need to maintain an equilibrium of surface diffusion fluxes in a system with a large number of degrees-of-freedom. The nanowire breaks up into drops on the left and right from the central part (see the blue rectangle in Fig. S7) for which 'time has almost stopped'.

We note that even if the central fragment breaks into two droplets, the break-up parameter, $\Lambda / r_{0}$, will take the known value in the final state: $\Lambda / r_{0} \approx 400 /(8 \times 5.5) \sim 9$ (as always, the first and the last fragments are counted as one drop due to pseudo-periodic boundary conditions). Whereas in the configuration depicted in subpart (b) of Fig. S7 the nanorod is divided by necks into eleven clearly visible fragments, i.e. $\lambda_{\max } / r_{0} \approx 6.6$ for the moment. This, once again, shows a tough competition between necks with the absorption of each other at the nonlinear break-up stage. 


\section{WILEY-VCH}

A

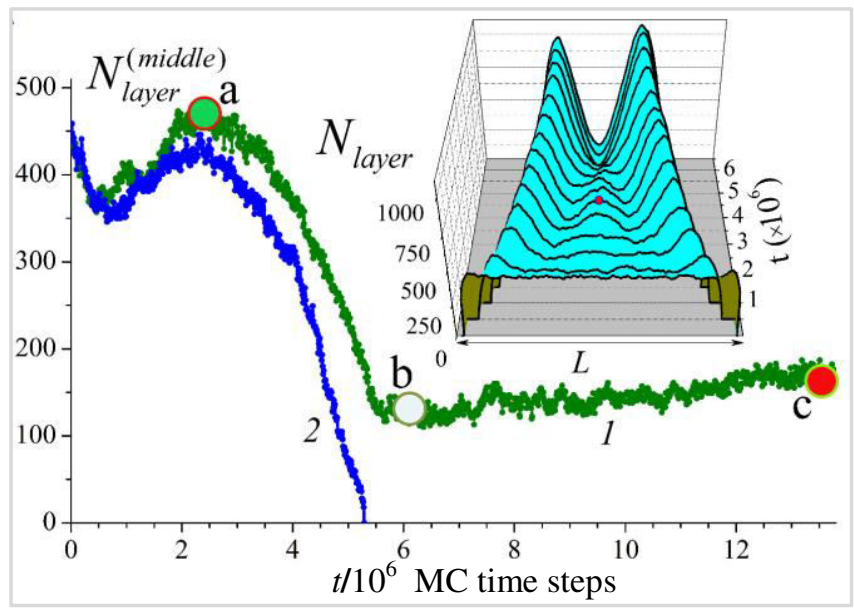

B

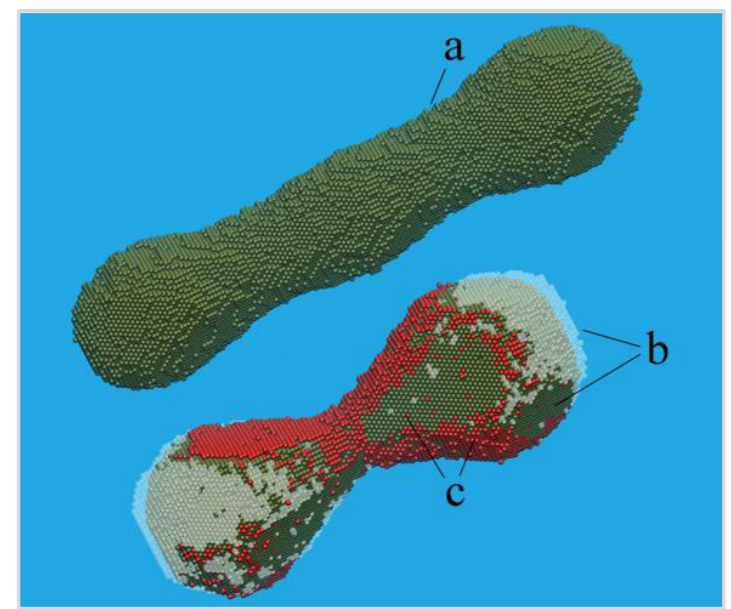

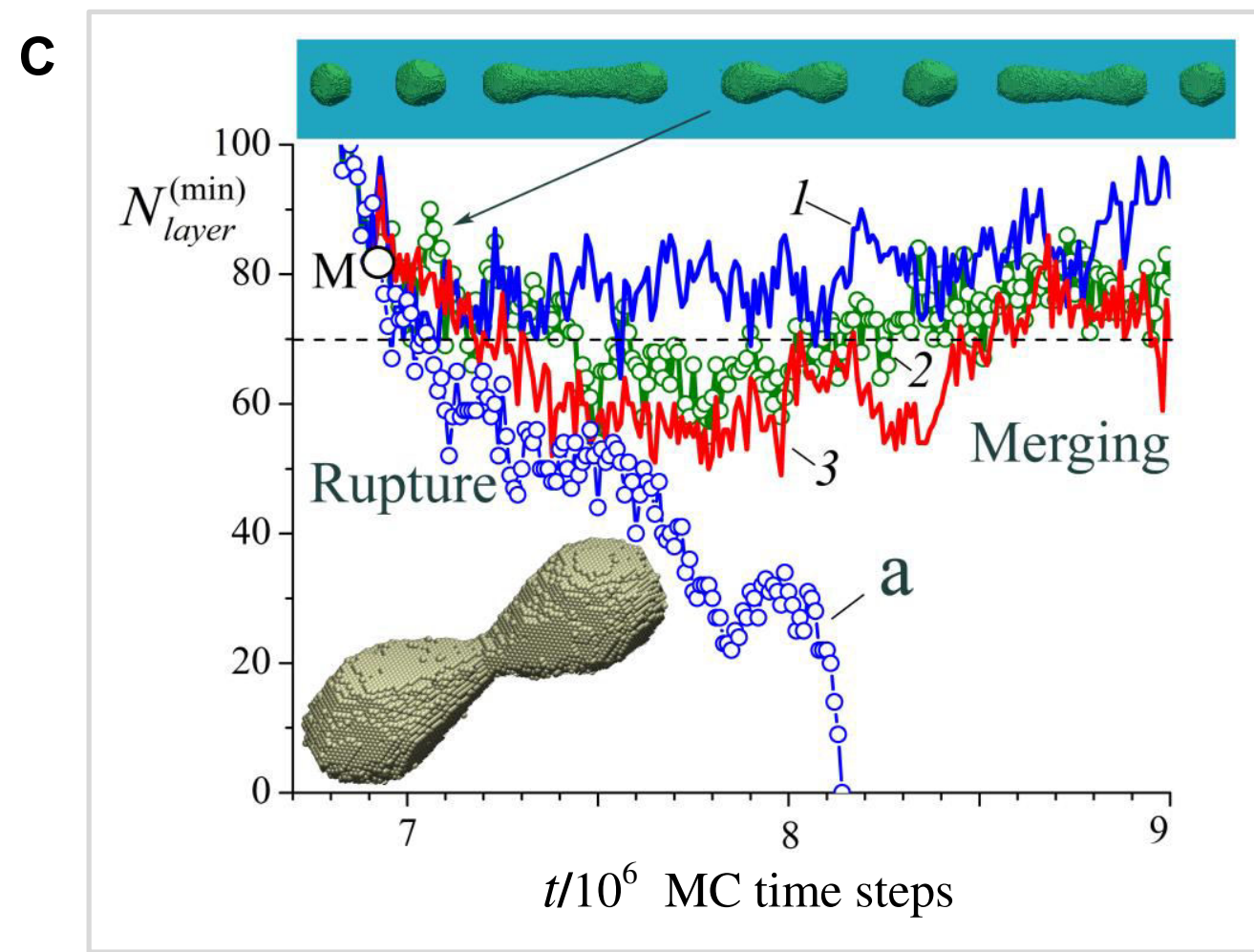

Fig. S6. Dynamics of the nanorod with [111]-type orientation. $L=120, d=15(L / d=8)$, warm regime: $\alpha=0.9, p=0.725$. The number of atoms in the nanowire equals $N_{0}=96200$. (A) Time dependence of the number of atoms $N_{\text {layer }}^{(\text {middle })}(t)$ in the central layer perpendicular to the nanowire axis (the result of two random simulations). In the inset, one can see how the distribution along the nanorod of the number of atoms in (111) layers changes with time (from $t=1 \times 10^{4}$ to $t=6.5 \times 10^{6}$ and with the time step $\Delta t=0.5 \times 10^{6}$ ). (B) Shape of the nanorod in the first simulation at $\mathrm{t}=2.5 \times 10^{6}$, when $N_{\text {layer }}^{\text {(middle) }}$ reaches its maximum (point (a) on the first (green) curve or red circle in the inset). The shapes of the nanorod at $t=6 \times 10^{6}$ and $t=13 \times 10^{6}$ are combined (corresponding to points (b) and (c) on the first curve). Olive atoms are common for both time moments; glass-like atoms (subpart b) and red atoms (subpart c) represent changes in the form of the nanorod with time. (C) Dynamics of a two-body dumbbell formed during the nanowire break-up and shown at the top and bottom insets: $t=6.9 \times 10^{6}, L=480, d=12, \alpha=1$, and $p=0.7$. The point $\mathrm{M}$ corresponds to the bifurcation state of the unduloid-like configuration with unpredictable evolution. 


\section{WILEY-VCH}

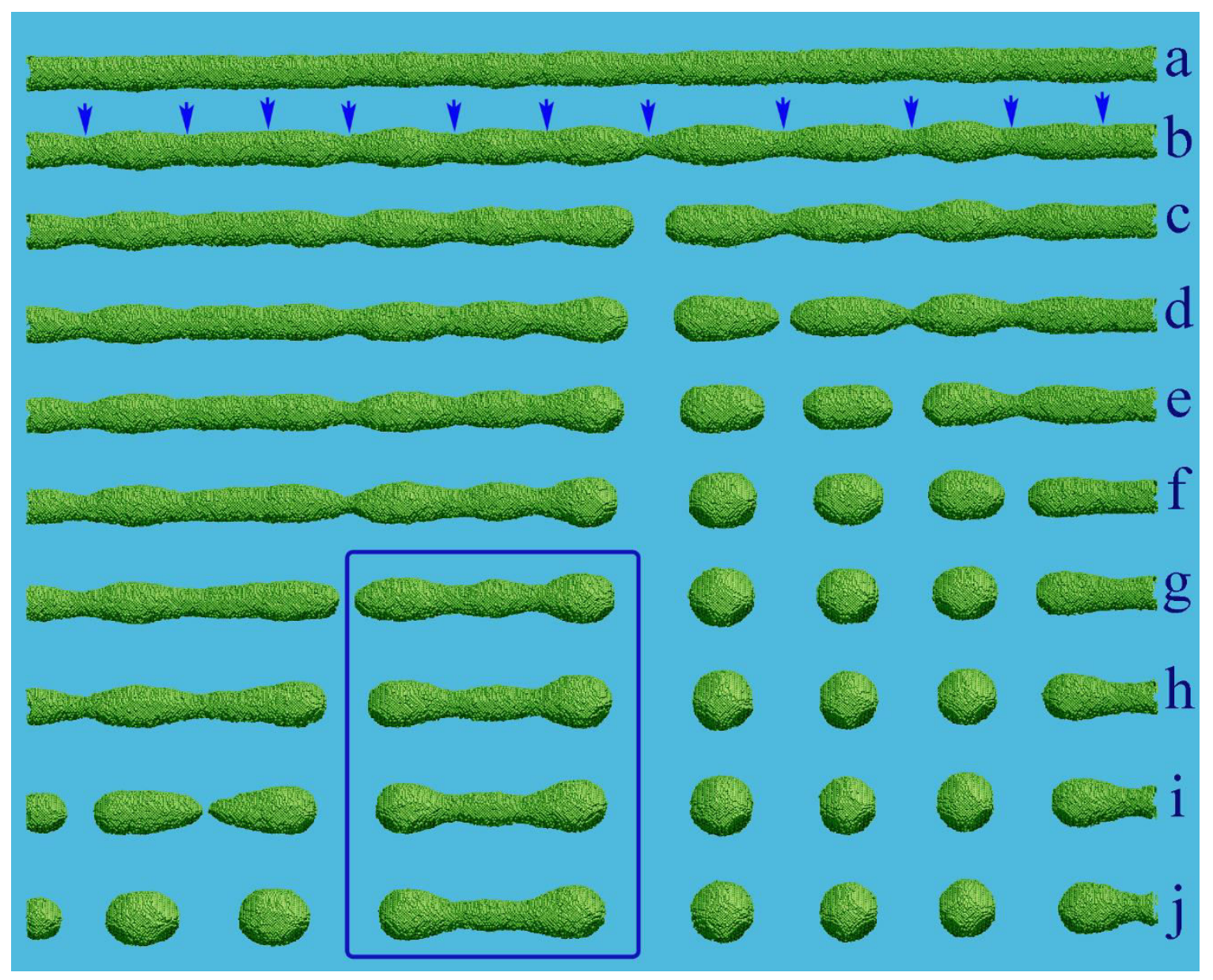

Fig. S7. Dynamics of the nanowire with [111]-type orientation in cold regime; $\alpha=1.2, p=0.65$, $d=11, L=400$, frozen ends, [100]-orientation, $N_{0}=165273$ atoms; in quasi-equilibrium state only, 1400 free atoms are around the nanowire. Subparts (a) to $(\mathrm{j}): \mathrm{t}=\{5,10,11,12,13,14.5,15.5,17,19$, 21\} $\times 10^{6}$ MC time steps. 